\title{
Epigenetic activation of meiotic recombination near Arabidopsis thaliana centromeres via loss of H3K9me2 and non-CG DNA methylation
}

\author{
Charles J. Underwood, ${ }^{1,2,3}$ Kyuha Choi, ${ }^{2,4}$ Christophe Lambing, ${ }^{2}$ Xiaohui Zhao, $^{2}$ \\ Heïdi Serra, ${ }^{2}$ Filipe Borges, ${ }^{1}$ Joe Simorowski, ${ }^{1}$ Evan Ernst, ${ }^{1}$ Yannick Jacob, ${ }^{1,5}$ \\ Ian R. Henderson, ${ }^{2}$ and Robert A. Martienssen ${ }^{1}$ \\ ${ }^{1}$ Howard Hughes Medical Institute, Watson School of Biological Sciences, Cold Spring Harbor Laboratory, Cold Spring Harbor, \\ New York 11724, USA; ${ }^{2}$ Department of Plant Sciences, University of Cambridge, Cambridge, CB2 3EA, United Kingdom
}

\begin{abstract}
Eukaryotic centromeres contain the kinetochore, which connects chromosomes to the spindle allowing segregation. During meiosis, centromeres are suppressed for inter-homolog crossover, as recombination in these regions can cause chromosome missegregation and aneuploidy. Plant centromeres are surrounded by transposon-dense pericentromeric heterochromatin that is epigenetically silenced by histone 3 lysine 9 dimethylation (H3K9me2), and DNA methylation in CG and non-CG sequence contexts. However, the role of these chromatin modifications in control of meiotic recombination in the pericentromeres is not fully understood. Here, we show that disruption of Arabidopsis thaliana H3K9me2 and non-CG DNA methylation pathways, for example, via mutation of the H3K9 methyltransferase genes KYP/SUVH4 SUVH5 SUVH6, or the CHG DNA methyltransferase gene CMT3, increases meiotic recombination in proximity to the centromeres. Using immunocytological detection of MLH1 foci and genotyping by sequencing of recombinant plants, we observe that H3K9me2 and nonCG DNA methylation pathway mutants show increased pericentromeric crossovers. Increased pericentromeric recombination in H3K9me2/ non-CG mutants occurs in hybrid and inbred backgrounds and likely involves contributions from both the interfering and noninterfering crossover repair pathways. We also show that meiotic DNA double-strand breaks (DSBs) increase in H3K9me2/ non-CG mutants within the pericentromeres, via purification and sequencing of SPOll-1-oligonucleotides. Therefore, H3K9me2 and non-CG DNA methylation exert a repressive effect on both meiotic DSB and crossover formation in plant pericentromeric heterochromatin. Our results may account for selection of enhancer trap Dissociation (Ds) transposons into the CMT3 gene by recombination with proximal transposon launch-pads.
\end{abstract}

[Supplemental material is available for this article.]

Eukaryotic centromeres are the sites of kinetochore attachment to spindle microtubules that allow chromosome segregation (McKinley and Cheeseman 2015). Centromere identity is governed by nucleosomes containing CENPA/CENH3-related histone variants, which occupy large arrays of tandemly repeated satellite sequences (Allshire and Karpen 2008; Malik and Henikoff 2009). A conserved feature of centromeres shared across eukaryotes is suppression of meiotic crossover (Lambie and Roeder 1988; Copenhaver et al. 1999; Vincenten et al. 2015; Nambiar and Smith 2016). Crossover suppression is important for fertility, as centromere-proximal recombination events have been associated with chromosome segregation errors and aneuploidy (Koehler et al. 1996; Lamb et al. 1996; Rockmill et al. 2006). Plants, animals, and fungi also typically possess repetitive pericentromeric heterochromatin, containing a high density of transposable elements (Allshire and Karpen 2008; Malik and Henikoff 2009; Nambiar and Smith 2016). In crop genomes, including wheat, barley,

\footnotetext{
Present addresses: ${ }^{3}$ Vegetable Crop Research, KeyGene, 6708 PW Wageningen, The Netherlands; ${ }^{4}$ Department of Life Sciences, Pohang University of Science and Technology, Pohang, Gyeongbuk, 37673, Republic of Korea; 5 Department of Molecular, Cellular \& Developmental Biology, Yale University, New Haven, CT 06511, USA Corresponding authors: irh25@cam.ac.uk, martiens@cshl.edu Article published online before print. Article, supplemental material, and publication date are at http://www.genome.org/cgi/doi/10.1101/gr.227116.117. Freely available online through the Genome Research Open Access option.
}

maize, and tomato, extensive pericentromeric heterochromatin occupies more than half of the chromosome and is also crossover-suppressed (Lhuissier et al. 2007; Wei et al. 2009; Mayer et al. 2012; Choulet et al. 2014). However, the genetic and epigenetic factors that shape meiotic recombination patterns in eukaryote centromeric and pericentromeric regions remain to be fully understood.

Arabidopsis thaliana centromeres consist of megabase tandem arrays of the 178- to 180-base pair CEN180 satellite repeat (Copenhaver et al. 1999; Kumekawa et al. 2000; Nagaki et al. 2003; Ito et al. 2007). The centromeric regions are also densely DNA methylated and enriched for $\mathrm{H} 3 \mathrm{~K} 9 \mathrm{me} 2$ and histone variant H2A.W (Lippman et al. 2004; Lister et al. 2008; Stroud et al. 2013; Yelagandula et al. 2014). Within the centromeric satellite arrays, a subset of repeats are occupied by nucleosomes containing the centromeric variant histone H3 CENH3 (Nagaki et al. 2003; Maheshwari et al. 2017). Surrounding the CEN180 satellite arrays are repetitive, transposon-dense regions of pericentromeric heterochromatin (Lippman et al. 2004; Lister et al. 2008; Stroud et al. 2013; Yelagandula et al. 2014). Plant transposable elements are transcriptionally silenced by $\mathrm{H} 3 \mathrm{~K} 9 \mathrm{me} 2$ and DNA cytosine methylation in $\mathrm{CG}$ and non-CG (CHG and $\mathrm{CHH}$, where $\mathrm{H}=\mathrm{A}, \mathrm{C}$, or $\mathrm{T})$

(C) 2018 Underwood et al. This article, published in Genome Research, is available under a Creative Commons License (Attribution 4.0 International), as described at http://creativecommons.org/licenses/by/4.0/. 
sequence contexts (Lippman et al. 2004; Du et al. 2012; Stroud et al. 2013, 2014). A. thaliana mutants that lose maintenance of CG or non-CG DNA methylation show elevated transposon transcription and mobility at high and moderate levels, respectively (Miura et al. 2001; Singer et al. 2001; Kato et al. 2003; Mirouze et al. 2009; Reinders et al. 2009; Teixeira et al. 2009; Tsukahara et al. 2012; Marí-Ordóñez et al. 2013; Stroud et al. 2014).

In $A$. thaliana, the chromodomain cytosine methyltransferases CHROMOMETHYLASE2 (CMT2) and CHROMOMETHYLASE3 (CMT3) recognize heterochromatic H3K9me2 via BAH and chromodomains, and methylate-associated DNA in $\mathrm{CHH}$ and CHG contexts, respectively (Bartee et al. 2001; Lindroth et al. 2001; Du et al. 2012; Stroud et al. 2013, 2014; Zemach et al. 2013; Dubin et al. 2015). Methylation of histone H3K9 requires the SET domain methyltransferases KRYPTONITE/SUPPRESSOR OF VARIEGATION HOMOLOG4 (KYP/SUVH4), SUPPRESSOR OF VARIEGATION HOMOLOG5 (SUVH5), and SUPPRESSOR OF VARIEGATION HOMOLOG6 (SUVH6), which are recruited to methylated DNA by SRA methyl-cytosine binding domains (Jackson et al. 2002; Malagnac et al. 2002; Ebbs and Bender 2006; Johnson et al. 2007; Stroud et al. 2013, 2014; Du et al. 2014). The de novo DNA methyltransferase DOMAINS REARRANGED METHYLTRANSFERASE2 (DRM2) is also required for maintenance methylation of non-CG contexts and thus can also affect H3K9me2 (Cao and Jacobsen 2002; Cao et al. 2003; Stroud et al. 2013, 2014). The kyp suvh5 suvh6 mutant abolishes almost all H3K9me2 and CHG/CHH methylation, but CG methylation remains intact, while cmt 3 mutants lose CHG methylation and $\mathrm{H} 3 \mathrm{~K} 9 \mathrm{me} 2$ is reduced, but $\mathrm{CHH}$ methylation and CG methylation are largely unchanged (Bartee et al. 2001; Lindroth et al. 2001; Jackson et al. 2002; Malagnac et al. 2002; Inagaki et al. 2010; Stroud et al. 2013, 2014; Yelagandula et al. 2014). In contrast to plants, in fission yeast, which lacks DNA methylation, the SET domain histone lysine methyltransferase CRYPTIC LOCI REGULATOR4 (CLR4) is recruited to methylated H3K9me2 directly via its chromodomain (Allshire and Ekwall 2015). Thus, by separating chromodomains (CMT2 and CMT3) from SET domains (KYP/ SUVH5/SUVH6), plants have introduced non-CG DNA methylation as an additional layer of epigenetic control underlying H3K9me2. Alongside these mechanisms, the METHYLTRANSERFERASE1 (MET1) cytosine methyltransferase, VARIANT IN METHYLATION1 (VIM1) family proteins, and the DECREASE IN DNA METHYLATION1 (DDM1) SWI/SNF chromatin remodeling protein are required for maintenance of CG context DNA methylation (Kankel et al. 2003; Saze et al. 2003; Lippman et al. 2004; Lister et al. 2008; Woo et al. 2008; Stroud et al. 2013). In this work, we use mutations in $A$. thaliana heterochromatic silencing pathways to investigate epigenetic control of meiotic recombination in the pericentromeric regions.

Meiotic crossovers form via inter-homolog repair of DNA double-strand breaks (DSBs) that are generated by SPO11 topoisomerase-related complexes (Szostak et al. 1983; Keeney et al. 1997; Robert et al. 2016; Vrielynck et al. 2016). Diverse eukaryotes have evidence for recombination hotspots, which are approximately kilobase-size regions with an elevated frequency of meiotic DSBs or crossovers, compared to the genome average or surrounding regions (Kauppi et al. 2004; De Massy 2013; Choi and Henderson 2015). Hotspots in different eukaryotic lineages are controlled to varying degrees by genetic and epigenetic information (Kauppi et al. 2004; De Massy 2013; Choi and Henderson 2015). At the chromosome-scale, A. thaliana crossover frequency is highest in gene-dense euchromatin, whereas the heterochro- matic centromeres are crossover-suppressed (Copenhaver et al. 1999; Giraut et al. 2011; Salomé et al. 2012; Yelina et al. 2015). At the fine-scale, plant crossover hotspots occur at gene promoters and terminators, and recombination is promoted by euchromatic modifications, including histone variant H2A.Z (Choi et al. 2013; Hellsten et al. 2013; Wijnker et al. 2013; Shilo et al. 2015). Acquisition of DNA methylation and H3K9me2 via the RNA-directed DNA methylation (RdDM) pathway is sufficient to silence A. thaliana euchromatic crossover hotpots (Yelina et al. 2015). This is consistent with DNA methylation suppressing meiotic DNA double-strand breaks in mouse (Zamudio et al. 2015), and silencing crossovers in Ascobolus (Maloisel and Rossignol 1998). Furthermore, loss of RNAi and Clr4-dependent H3K9me2 elevates centromeric crossovers in fission yeast (Ellermeier et al. 2010), and Drosophila position effect variegation (PEV) suppressor mutations (Suppressor of Variegation) can modify centromeric crossover frequency (Westphal and Reuter 2002). Interestingly, the $A$. thaliana met1 and $d d m 1$ CG methylation mutants associate with remodeling of meiotic recombination along chromosomes, with crossover increases in the chromosome arms and decreases across the pericentromeres (Colomé-Tatché et al. 2012; Melamed-Bessudo and Levy 2012; Mirouze et al. 2012; Yelina et al. 2012, 2015). However, how non-CG DNA methylation and other epigenetic silencing pathways contribute to recombination landscapes along plant chromosomes has not been fully explored. In this study, we address the roles of H3K9me2 and non-CG DNA methylation in suppression of meiotic DSBs and crossovers within A. thaliana pericentromeric heterochromatin.

\section{Results}

\section{Epigenetic activation of pericentromeric crossovers in non-CG/H3K9me2 mutants}

To investigate meiotic recombination frequency in $A$. thaliana non-CG DNA methylation and H3K9me2 mutants, we used fluorescent crossover reporter lines (fluorescent tagged lines, FTLs) (Melamed-Bessudo et al. 2005; Berchowitz and Copenhaver 2008). FTLs express different colors of fluorescent protein under seed (NapA)- or pollen (LAT52)-specific promoters, from linked T-DNAs insertions (Fig. 1A). The scoring of fluorescent color inheritance in the progeny seed or pollen (male gametes) of FTL hemizygotes allows the measurement of sex-averaged or male-specific crossover frequency, respectively, in defined chromosomal intervals (Fig. 1A; Melamed-Bessudo et al. 2005; Berchowitz and Copenhaver 2008; Yelina et al. 2013).

We first analyzed crossover frequency within the 5.4-megabase $(\mathrm{Mb})$ CEN3 FTL interval, which spans the centromere and pericentromeric heterochromatin of Chromosome 3 , in wild type versus non-CG/H3K9me2 pathway mutants (Fig. 1B; Yelina et al. 2015). Genetic ablation of the H3K9 methyltransferases (kyp suvh5 suvh6) or the non-CG DNA methyltransferases (drm1 drm2 cmt2 cmt3) eliminates both H3K9me2 and non-CG DNA methylation, while single and double mutants have intermediate effects (Cao et al. 2003; Stroud et al. 2013, 2014). We observed that mutations that disrupt H3K9me2 and non-CG DNA methylation to progressively greater extents resulted in progressively greater increases in CEN3 crossover frequency $(\mathrm{drm} 1 \mathrm{drm} 2<k y p<$ cmt3 < drm1 drm2 cmt3 < kyp suvh5 suvh6; all $\chi^{2} P<2.0 \times 10^{-16}$ ) (Fig. 1C; Supplemental Table S1; Stroud et al. 2013, 2014). The suvh 5 suvh 6 and cmt 2 mutants did not show significant differences compared to wild type (Fig. 1C; Supplemental Table S1).

\section{Genome Research}

www.genome.org 
A

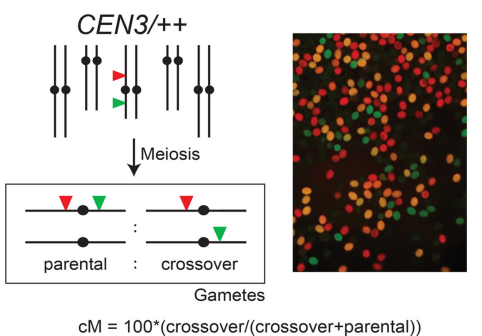

B

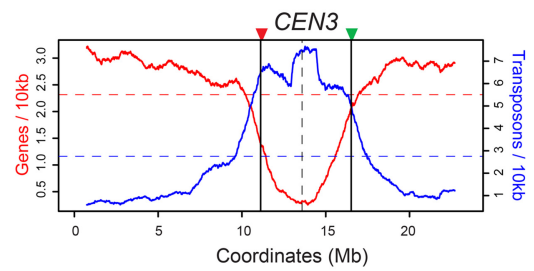

D

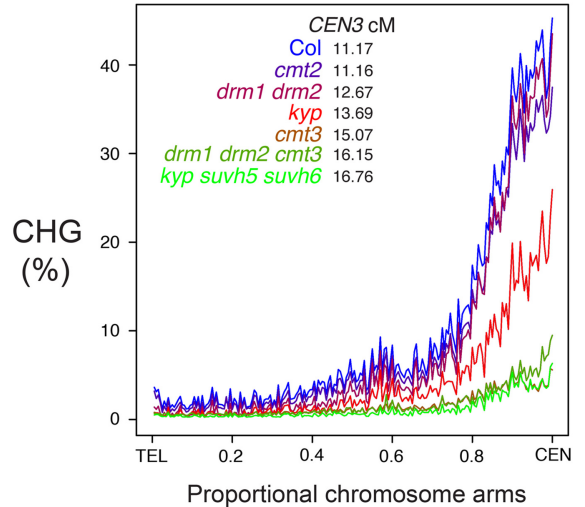

C

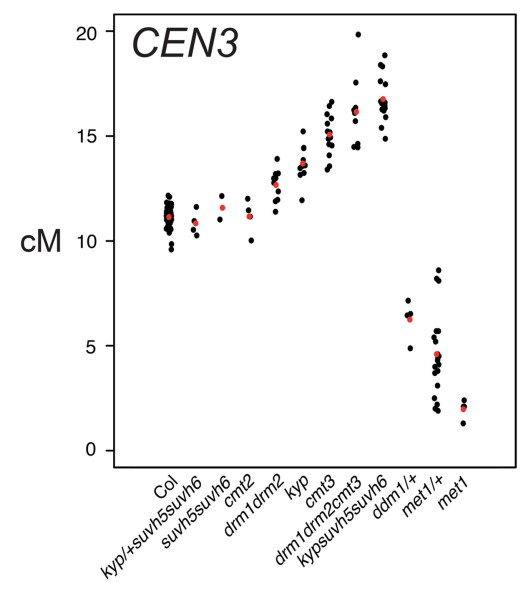

E

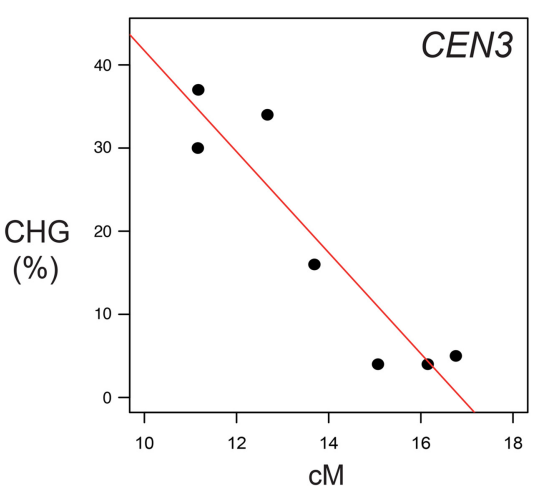

Figure 1. Progressive increases of pericentromeric crossover frequency in $\mathrm{H} 3 \mathrm{~K} 9 \mathrm{me} 2$ and non-CG DNA methylation pathway mutants. $(A)$ Measurement of crossover frequency using segregation of hemizygous fluorescent tagged line (FTL) T-DNAs. A representative fluorescent micrograph is shown of $F T L /+$ pollen, reproduced from Choi et al. (2016). (B) A rolling mean of gene (red) and transposon (blue) density (number of start coordinates per adjacent 10-kb window) are plotted along Chromosome 3 , with the location of CEN3 FTL T-DNAs indicated by vertical lines. Mean values are indicated by the horizontal dotted lines and the centromere assembly gap by the vertical dotted line. (C) CEN3 crossover frequency (cM) in DNA methylation mutants. Data for met1 and met1/+ are reproduced from Yelina et al. (2015). Black dots represent replicate measurements and red dots show mean values. (D) Published BS-seq data (Stroud et al. 2013) were used to analyze CHG DNA methylation density along chromosome telomere-centromere axes in wild type and $\mathrm{H} 3 \mathrm{~K} 9 \mathrm{me} 2$ /non-CG DNA methylation mutants. Lines are colored according to $C E N 3 \mathrm{cM}$ (blue=highest, red=intermediate, green = lowest). (E) Correlation between CEN3 CM and CHG DNA methylation from published BS-seq data (Stroud et al. 2013).

We used published bisulfite sequencing data to analyze DNA methylation levels within the CEN3 interval in the genotypes analyzed for crossover frequency (Stroud et al. 2013). Within this series of mutants, levels of CHG DNA methylation showed a strong negative correlation with CEN3 genetic distance (Pearson's $r=$ $-0.93, P=2.36 \times 10^{-3}$ ), whereas CG and CHH methylation were not significantly correlated (Fig. 1D,E; Supplemental Table S2; Stroud et al. 2013). In contrast to non-CG/H3K9me2 mutants, heterozygous $d d m 1 /+, m e t 1 /+$, or homozygous met 1 mutants, which inherit chromosomes hypomethylated in the CG context, have reduced CEN3 recombination, as reported previously (Fig. 1C; Supplemental Table S1; Colomé-Tatché et al. 2012; MelamedBessudo and Levy 2012; Mirouze et al. 2012; Yelina et al. 2012, 2015). We confirmed release of crossover suppression in cmt 3 across the Chromosome 5 centromere and pericentromeric heterochromatin, using additional FTLs in Col (CTL5.11, $\chi^{2} \quad P=1.30 \times 10^{-3}$ ) and Ler (LTL5.4, $\left.\chi^{2} P=2.09 \times 10^{-9}\right)$ inbred backgrounds (Supplemental Fig. S1; Supplemental Tables S3, S4). We also crossed cmt3 alleles in Col (cmt3-11) and Ler (cmt3-7) accessions together to generate $\mathrm{Col} / \mathrm{Ler} \mathrm{F}_{1}$ progeny that were $\mathrm{cmt} 3$ mutant and carried the CEN3 FTL (Fig. 2A; Lindroth et al. 2001). We observed significantly increased CEN3 genetic distance in $c m t 3$ hybrids $\left(\chi^{2} P=1.27 \times 10^{-86}\right)$, similar to the increase observed for inbreds (Fig. 2B; Supplemental Tables S1, S5). In contrast, recombination in the euchromatic 420 FTL interval on Chromosome 3 did not significantly change in $\mathrm{cmt} 3$ inbreds and slightly decreased in hybrids $\left(\chi^{2} P=\right.$ $1.96 \times 10^{-3}$ ), compared to wild type (Fig. 2C; Supplemental Table S6). Together, these data indicate that mutations in the H3K9me2/non-CG pathway primarily activate crossover frequency in proximity to the centromeres.

\section{Genome-wide mapping of crossovers in chromomethylase3 mutants}

We next sought to map crossovers genome-wide in wild type compared with a H3K9me2/non-CG mutant background, using segregation of single nucleotide polymorphisms (SNPs). We used cmt3 mutant alleles in both Col (cmt311) and Ler (cmt3-7) backgrounds (Bartee et al. 2001; Lindroth et al. 2001; Stroud et al. 2013, 2014) to generate wild type $(\mathrm{Col} \times \mathrm{Ler})$ and $\mathrm{cmt} 3(\mathrm{cmt} 3$ $11 \times c m t 3-7) \mathrm{F}_{2}$ populations of $>700$ individuals each (Fig. 2A). To assess centromeric recombination levels in these populations, we genotyped $\mathrm{Col} / \mathrm{Ler}$ simple sequence length polymorphism (SSLP) markers on Chromosomes 1 and 3 (Supplemental Table S7). This confirmed significant increases in pericentromeric recombination in the cmt3 population compared to wild type, consistent with our previous FTL measurements (Supplemental Table S7). To map crossovers at high resolution, we performed genotyping by sequencing (GBS) of 437 wild-type and $384 \mathrm{cmt} 3 \mathrm{~F}_{2}$ individuals, which identified 3320 and 2803 crossovers, respectively (Fig. 2D-F; Supplemental Table S8; Rowan et al. 2015; Choi et al. 2016; Serra et al. 2018). The crossovers were mapped between Col/Ler SNPs to a mean resolution of $887 \mathrm{bp}$. The total number of crossovers per wild-type $F_{2}$ individual (mean $=7.6$ ) was comparable to that observed in similar $\mathrm{F}_{2}$ populations (Giraut et al. 2011; Salomé et al. 2012) and was not significantly different in cmt3 (mean $=7.3$; Mann-WhitneyWilcoxon test, $P=0.101$ ) (Fig. 2E; Supplemental Table S8).

To analyze crossover distributions throughout the genome, we defined centromeres as the crossover-suppressed regions that surround gaps in the chromosome assembly (Copenhaver et al. 1999; Kumekawa et al. 2000), the pericentromeres as the 
A
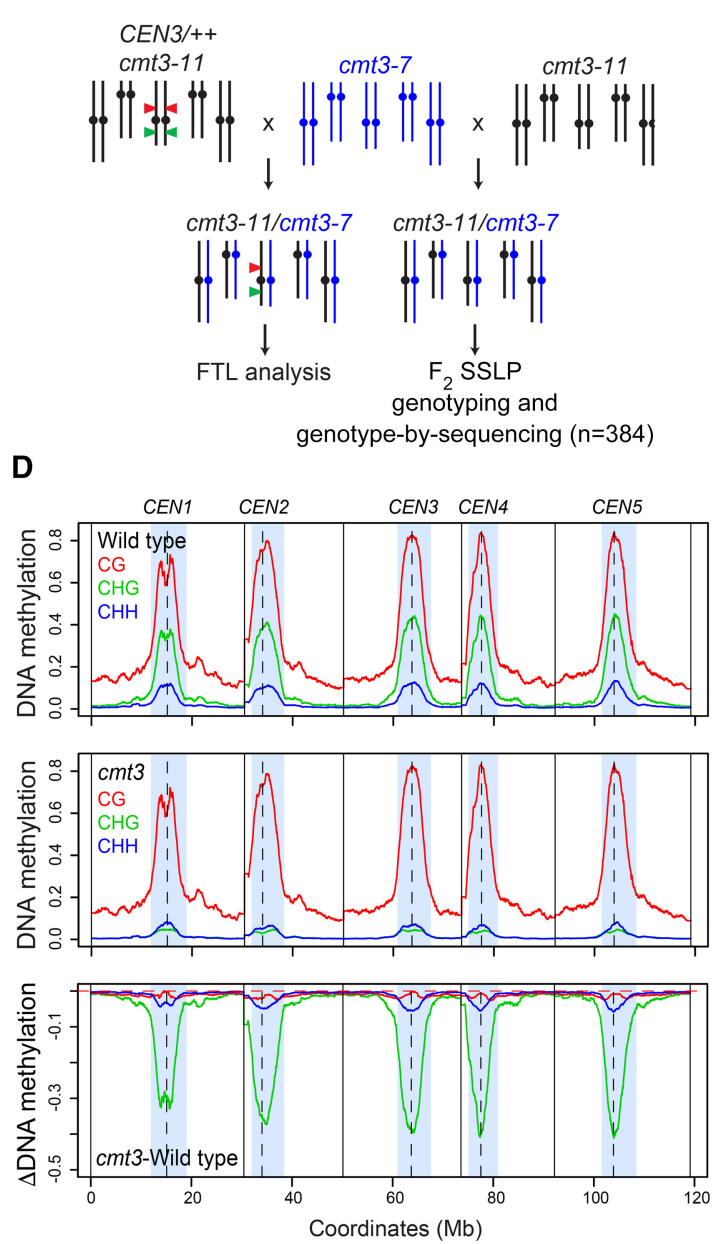

E
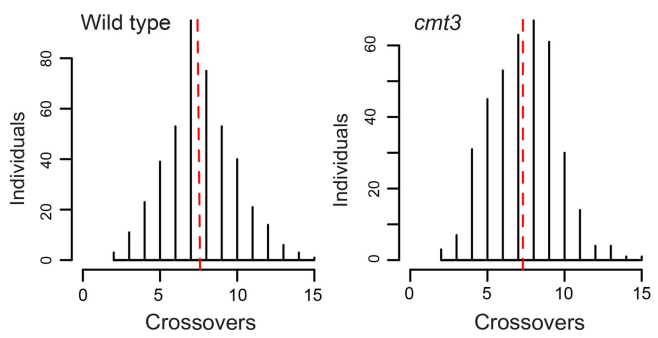

B

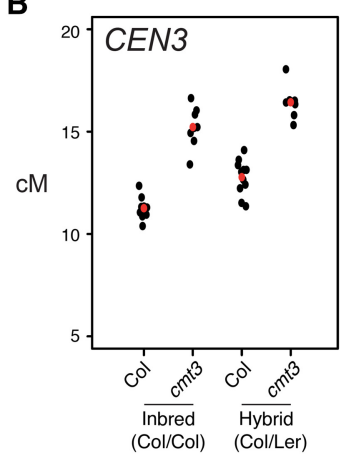

C

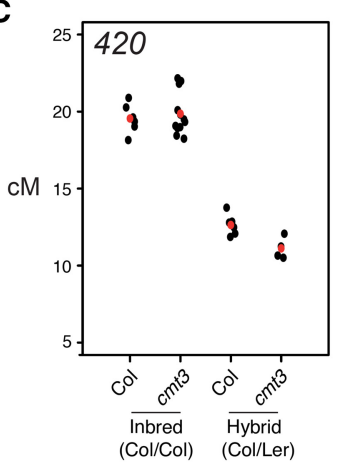

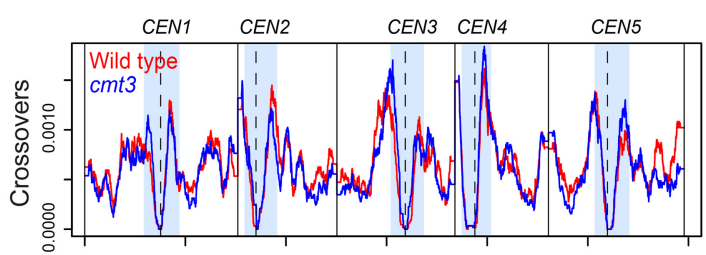
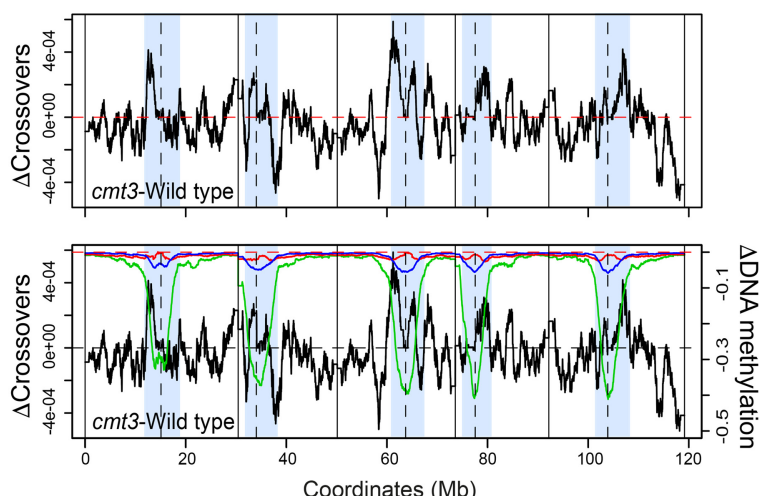

$\mathbf{F}$

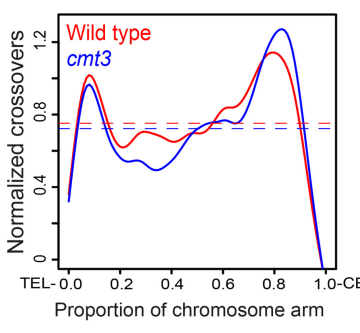

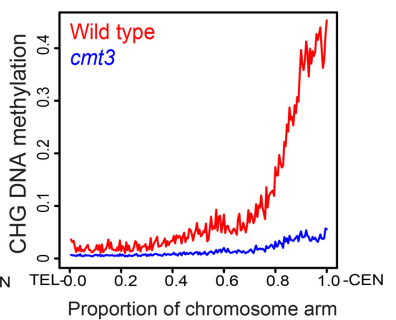

Figure 2. Genome-wide mapping of crossover frequency in $\mathrm{cm} t 3$ non-CG mutants. ( $A$ ) Crossing scheme used to analyze recombination in $\mathrm{cm} t 3 \mathrm{mutants}$. Col chromosomes are black and Ler chromosomes are blue. The CEN3 FTL T-DNAs are indicated by red and green triangles. (B) CEN3 crossover frequency (cM) in wild type and $\mathrm{cmt} 3$, in Col/Col inbreds, or Col/Ler $\mathrm{F}_{1}$ hybrids. Replicate measurements are shown in black and mean values in red. (C) $420 \mathrm{crossover}$ frequency in wild type and $c m t 3$, in Col/Col inbreds, or Col/Ler $\mathrm{F}_{1}$ hybrids, as shown for $B$. (D) Plots of the $A$. thaliana chromosomes on a continuous $x$-axis are shown. Analysis of DNA methylation frequency in CG (red), CHG (green), and CHH (blue) from published data in wild type (Col) or $\mathrm{cmt3-11}$ (Stroud et al. 2013) (left panels). A differential ( $\mathrm{cmt} 3$ - wild type $=\Delta$ DNA methylation) plot is also shown. Vertical dotted lines indicate the position of the centromere assembly gaps and vertical solid lines indicate telomeres. The pericentromeres, defined by higher than average DNA methylation, are indicated by light blue shading. The normalized frequency of crossovers mapped by GBS in wild type (red) and cmt 3 (blue) $\mathrm{F}_{2}$ populations is plotted (right panels), in addition to the $\mathrm{cmt} 3$ - wild type differential ( $\Delta$ Crossovers). (E) Histograms of crossovers per $\mathrm{F}_{2}$ individual for wild-type and $\mathrm{cm} t 3$ populations. Red dotted lines indicate mean values. ( $F$ ) Normalized crossover frequency analyzed along chromosome telomere (TEL) to centromere (CEN) axes in wild-type (red) and $\mathrm{cm}$ t3 (blue) populations. CHG DNA methylation was analyzed and plotted similarly for wild type (red) or $\mathrm{cmt3}$ (blue) (Stroud et al. 2013).

contiguous regions flanking the centromeres with higher than average DNA methylation, and chromosome arms as the remainder of the genome (Supplemental Table S9). Consistent with our FTL analysis, we observed that GBS-mapped crossovers were significantly increased in the cmt 3 pericentromeric regions $(24.6 \%$ versus
$27.8 \%$ of events were pericentromeric in wild type versus $\mathrm{cmt} 3, \chi^{2}$ $P=5.60 \times 10^{-3}$ ), which are strongly depleted of CHG DNA methylation in cmt3 (Fig 2D,F; Supplemental Table S10; Stroud et al. 2013). We also observed elevated centromeric crossovers $(n=13)$ in cmt3 $\left(\chi^{2} P=2.63 \times 10^{-4}\right)$, which were completely absent in

\section{Genome Research}

www.genome.org 
wild type (Supplemental Table S10). The chromosome arms showed a significant decrease of crossovers in cmt3 $\left(\chi^{2} P=1.50 \times\right.$ $10^{-3}$ ) (Supplemental Table S10). These data confirm that crossovers increase in proximity to cmt 3 centromeres, but that the increase is strongest in the flanking pericentromeric regions (Fig. $2 \mathrm{D}, \mathrm{F})$. However, we note that some pericentromeric regions showed higher crossover frequency in wild type than $\mathrm{cmt} 3$-for example, the right pericentromere of Chromosome 2-and therefore, region-specific effects also likely play an important role, such as structural genetic variation.

Meiotic immunocytology of chromatin and recombination in non-CG/H3K9me2 mutants

To assess H3K9me2 patterns during meiosis, we performed immunocytological staining using antibodies against this histone mod-
A
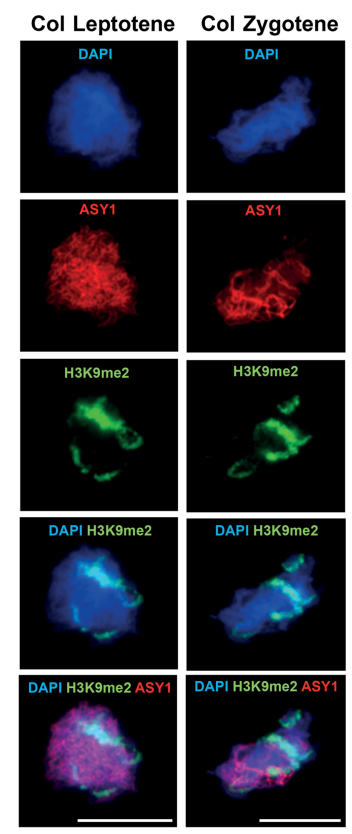

C
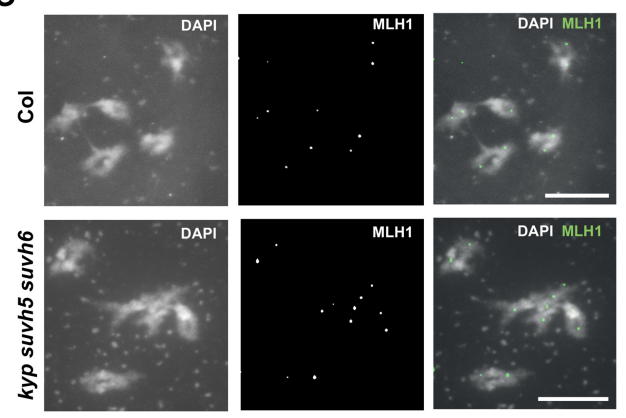

B
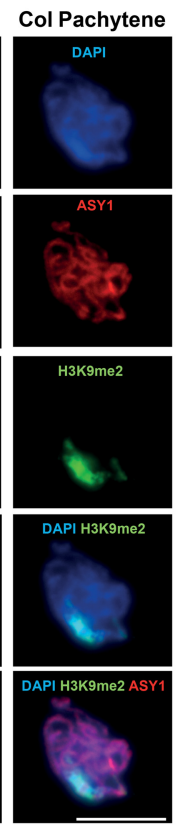
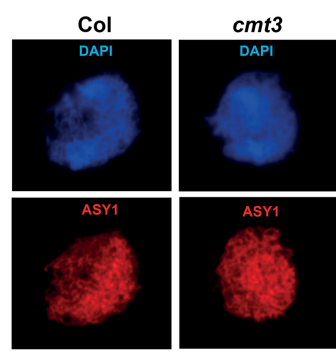

kyp suvh5 suvh6
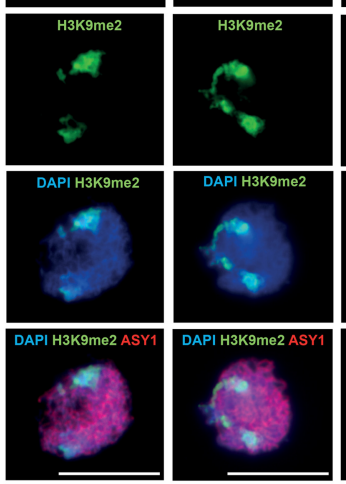

D

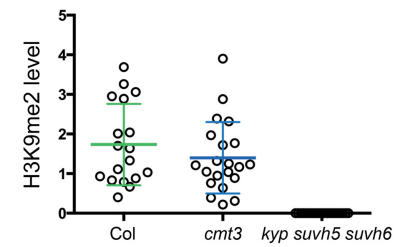

E

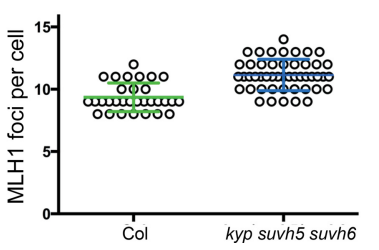

Figure 3. Meiotic heterochromatin is enriched for $\mathrm{H} 3 \mathrm{~K} 9 \mathrm{me} 2$ and shows increased MLH1 foci in kyp suvh5 suvh6. (A) Wild-type (Col) male meiocytes immunostained for ASY1 (red) and H3K9me2 (green) and stained for DNA (DAPI, blue), at leptotene, zygotene, and pachytene stages. Scale bar, $10 \mu \mathrm{M}$. (B) As for $A$, but showing leptotene stage cells from wild type (Col), cmt3-11, and kyp suvh5 suvh6. (C) Male meiocytes at diakinesis stained for DAPI and immunostained for MLH1 (green) in wild type and kyp suvh5 suvh6. Bivalent chromosomes are evident that are associated with MLH1 foci at crossover sites. (D) Quantification of $\mathrm{H} 3 \mathrm{~K} 9 \mathrm{me} 2$ immunostaining in wild type (Col), cmt3, and kyp suvh5 suvh6 meiotic cells. (E) Quantification of MLH1 foci in wild type and kyp suvh5 suvh6. ification and the chromosome axis HORMA domain protein ASYNAPTIC1 (ASY1) (Fig. 3A; Armstrong et al. 2002). During meiosis, $A$. thaliana centromeres and pericentromeres undergo pro(Fig. 3A; Armstrong et al. DAPI staining of DNA, stain strongly for $\mathrm{H} 3 \mathrm{~K} 9 \mathrm{me} 2$ throughout (Fig. 3A), which are the key occur (Armstrong et al. 2001; Sanchez-Moran et al. 2007). At suvh6 (Mann-Whitney-Wilcoxon test, $P=7.47 \times 10^{-9}$ ). Although the mean H3K9me2 signal was lower in cmt3 compared to wild3B,D; Supplemental Fig. S2; Supplemental Tables S11, S12), consistent with intermediate reductions in $\mathrm{H} 3 \mathrm{~K} 9 \mathrm{me} 2$ observed previously in cmt3 somatic cells (Inagaki et al. 2010; Yelagandula et al. 2014). Thus, H3K9me2 accumulates strongly in $A$. thaliana heterochromatin during meiosis, and its loss or reduction results in increased pericentromeric crossover.

Crossovers can be detected by immunostaining for MLH1, which marks class I interfering crossover foci (Crismani et al. 2012). Therefore, we scored MLH1 foci associated with euchromatin or heterochromatin, based on DAPI staining, in wild type and kyp suvh5 suvh6 mutants (Fig. 3C,E; Supplemental Table S13). We observed a slight but significant increase in MLH1 foci numbers in $k y p$ suvh5 suvh6 $($ mean $=11.1)$ compared to wild type (mean $=9.4)$ at diakinesis (Mann-Whitney-Wilcoxon test, $P=$ $1.64 \times 10^{-7}$ ) (Fig. 3C,E; Supplemental Table S13). Importantly, MLH1 foci were also significantly increased in $k y p$ suvh5 suvh6 heterochromatin (mean = 2.9 ), compared to wild type (mean $=1.7$; Mann-Whitney-Wilcoxon test, $P=$ $4.22 \times 10^{-5}$ ) (Fig. 3C,E; Supplemental Table S14). This provides cytological support for our crossover mapping data and indicates that MLH1-dependent repair contributes to the increase in pericentromeric crossovers observed in $\mathrm{H} 3 \mathrm{~K} 9 \mathrm{me} 2 /$ non-CG DNA methylation mutants.

As MLH1 foci were increased in $k y p$ suvh5 suvh6, we further investigated the relationship of class I and class II crossover pathways to the observed recombination changes. Approximately $85 \%$ of A. thaliana crossovers are dependent on the class I repair pathway and are interference-sensitive (Higgins et al. 2004; Mercier et al. 2005). Mutants in the class I pathway, for example, zip4, cause a strong reduction in crossovers and fertility (Mercier et al. 2005; Chelysheva et al. 2007). The fancm mutation restores fertility in fancm zip4 double mutants by 
increasing class II noninterfering crossovers (Crismani et al. 2012). Therefore, we constructed cmt3 zip4 and $\mathrm{cmt} 3$ fancm double mutants and compared CEN3 crossover frequency and fertility to wild type and single mutants (Fig. 4A-C; Supplemental Tables S14, S15). Unlike fancm, cmt3 was unable to suppress zip4 infertility (Fig. 4A,B; Supplemental Table S15). However, a small but significant CEN3 crossover increase was observed in cmt3 zip4, compared with zip4 alone $\left(\chi^{2} P=2.99 \times 10^{-9}\right)$, which indicates that the class II pathway may contribute to increased crossovers in cmt3 mutant centromeres (Fig. 4C; Supplemental Table S14). Additionally, the cmt3 fancm double mutant shows an additive increase in CEN3 crossover frequency, compared with $\mathrm{cmt} 3\left(\chi^{2} P=\right.$ $\left.3.34 \times 10^{-10}\right)$ and fancm $\left(\chi^{2} P=2.66 \times 10^{-7}\right)$ single mutants (Fig. $4 \mathrm{C}$; Supplemental Table S14). From these data, we conclude that increased pericentromeric crossovers in $\mathrm{H} 3 \mathrm{~K} 9 \mathrm{me} 2$ and non-CG DNA methylation mutants may involve contributions from both interfering and noninterfering repair pathways.

\section{Meiotic DSBs are elevated in the pericentromeres of non-CG/ H3K9me2 mutants}

During $A$. thaliana meiosis, SPO11-1 acts with SPO11-2 and MTOPVIB to generate DSBs, which can undergo inter-homolog repair to form crossovers (Grelon et al. 2001; Hartung et al. 2007; Vrielynck et al. 2016). SPO11 enzymes are related to topoisomerase-VI transesterases and become covalently bound to $\sim 20$ - to 50-base target site oligonucleotides during DSB formation (Keeney and Kleckner 1995; Pan et al. 2011; Lange et al. 2016). We have purified and sequenced $A$. thaliana SPO11-1-oligos in order to map patterns of meiotic DSBs genome-wide, using a complementing SPO11-1-Myc spo11-1 line (Choi et al. 2018). In order to profile meiotic DSBs in H3K9me2/non-CG DNA methylation mutants, we generated a SPO11-1-Myc spo11-1 kyp suvh5 suvh6 line and used this to purify and sequence SPO11-1-oligos (Supplemental Table S16). The coverage of combined unique and multiple mapped SPO11-1-oligo reads were normalized by library size. Replicate libraries showed significant correlation at multiple scales (Supplemental Table S17). For example, Pearson's $r$ values between replicate libraries at the 10 -kb scale were between 0.91 and 0.99 (Supplemental Table S17). Further normalization was performed using a single-ended Col genomic DNA library with reads trimmed to 50 base pairs, which were aligned as for SPO11-1-oligos, and then used to calculate $\log _{2}$ (SPO11-1-oligo/ gDNA) values. Finally, $z$-score standardization was applied, such that scores represent the signed number of standard deviations from the mean, and these values were used for downstream analysis.

To analyze chromosome-scale patterns, SPO11-1-oligo levels were calculated in 10-kb adjacent windows and plotted along the chromosomes using a rolling average. We also calculated a SPO11-1-oligo differential $(\Delta)$ by subtracting wild-type from $k y p$ suvh 5 suvh 6 values. We observed that the centromeric and pericentromeric regions showed a striking increase in SPO11-1-oligos in kyp suvh5 suvh6 (Fig. 5A,B). We similarly calculated mean CG, $\mathrm{CHG}$, and $\mathrm{CHH}$ methylation levels in 10-kb windows and calculated kyp suvh5 suvh6 differential values in the same way, using published data (Stroud et al. 2013). The kyp suvh5 suvh6 mutant shows strong reduction of $\mathrm{CHG}$ and $\mathrm{CHH}$ methylation in the pericentromeric regions, which was significantly correlated with the SPO111-oligo $\Delta$ differential (Pearson's CHG $r=-0.751, P<2.2 \times 10^{-16}$, CHG $r=-0.737, P=<2.2 \times 10^{-16}$ ) (Fig. 5A,B). The increase in SPO11-1-oligos in kyp suvh5 suvh6 occurred more strongly within the genetically defined centromeres, compared with the crossover changes in $\mathrm{cmt} 3$, which occurred most strongly within the adjacent pericentromeres (Figs. 2D,F, 5A,B). Hence, while both meiotic DSBs and crossovers increase in H3K9me2/non-CG mutant heterochromatin, they were elevated in adjacent centromeric and pericentromeric regions, respectively.

We next analyzed DSB frequency around copies of the CEN180 satellite repeat, which are found in proximity to the centromeres (Fig. 5C). Each A. thaliana chromosome sequence contains a centromere gap, which contains megabase arrays of CEN180 repeats (Copenhaver et al. 1999; Kumekawa et al. 2000; Ito et al. 2007; Maheshwari et al. 2017). Further matches to the CEN180 consensus flank these gaps, and we identified 3397 repeats in the Col reference genome that generally occur in tandemly repeated arrays (Fig. 5C). SPO11-1-oligo density was analyzed in 20$\mathrm{kb}$ windows around these repeat positions and compared to the same number of randomly chosen windows, in wild type and $k y p$ suvh5 suvh6 (Fig. 5D). Consistent with the SPO11-1-oligo increase in centromeric regions at the chromosome scale (Fig. 5A), we observed a pronounced increase in SPO11-1-oligo density within the CEN180 repeats at the fine-scale in kyp suvh5 suvh6 (Fig. 5D). The pattern of SPO11-1-oligos within the CEN180 repeat units is also altered in kyp suvh5 suvh6 (Fig. 5D). Together, this shows that meiotic DSBs increase in the CEN180 repeats in H3K9me2 mutant backgrounds. A relatively small number (141) of transposons are transcriptionally up-regulated in kyp suvh5 suvh6 mutants and associated with decreased non-CG methylation (Stroud et al. $2012,2013)$. Some of these elements were associated with elevated SPO11-1-oligonucleotides in kyp suvh5 suvh6 mutants, but others were not (Supplemental Fig. S3), indicating that transcriptional activation is not strictly coupled to up-regulation of meiotic SPO11-1-oligo formation at A. thaliana transposons.

Transposon insertions into CMT3 appear to induce meiotic recombination

In a large scale screen for de novo insertions of the nonautonomous maize transposable element Dissociation (Ds) introduced into $A$. thaliana, a transgene strongly expressing the Activator (Ac) transposase was crossed to plants
Figure 4. Genetic interactions between class I and class II crossover pathways and H3K9me2/non-CG methylation. (A) Silique length in wild type (Col), zip4, cmt3, and cmt3 zip4. (B) Seed set per silique in wild type (Col), zip4, cmt3, and cmt3 zip4. Replicate measurements are shown in black and mean values in red. (C) CEN3 crossover frequency (cM) in wild type, cmt3, zip4, and fancm mutant backgrounds. Replicate measurements are shown in black and mean values in red.

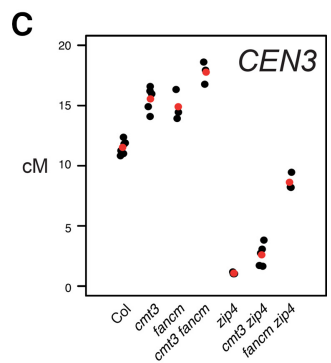

\section{Genome Research}

www.genome.org 
A
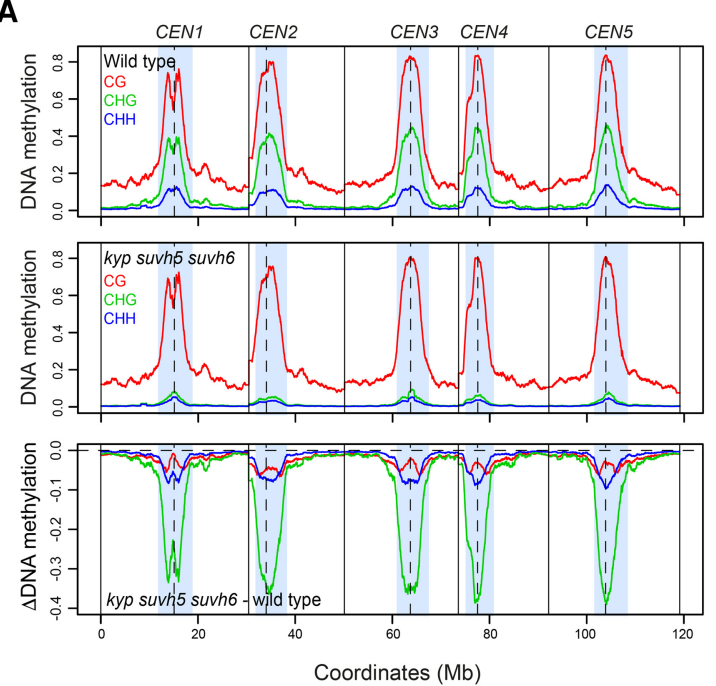

C
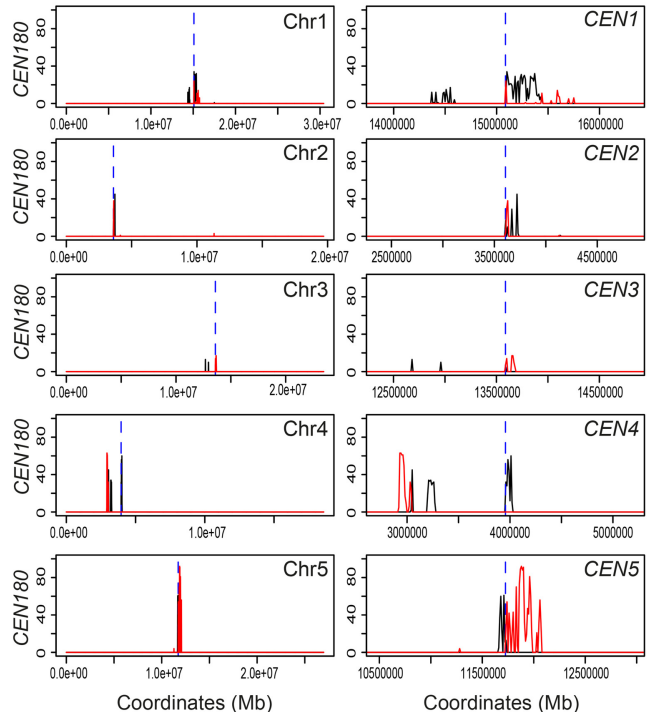

B
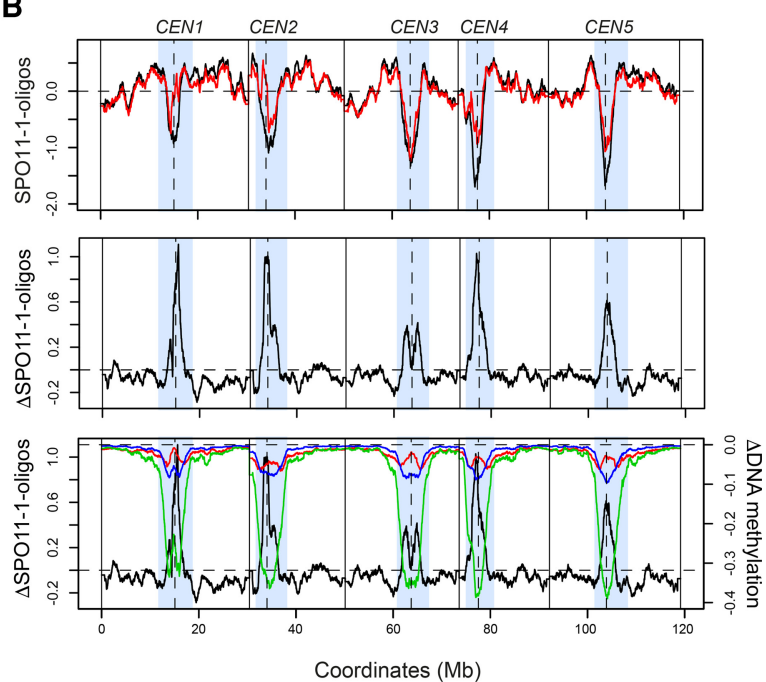

D
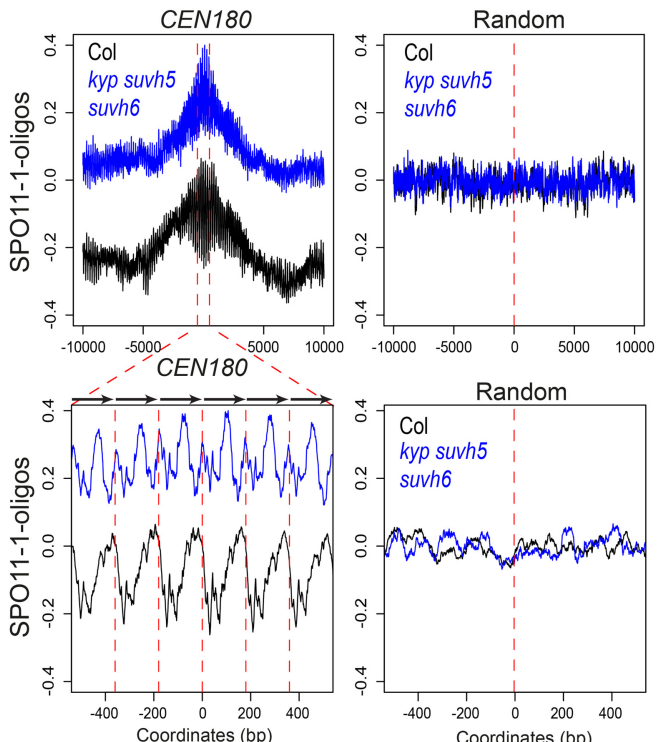

Figure 5. Elevated SPO11-1-oligonucleotides levels in centromeres of kyp suvh5 suvh6 H3K9me2 mutants. ( $A$ ) Plots of the $A$. thaliana chromosomes on a continuous $x$-axis are shown. Analysis of DNA methylation frequency in $\mathrm{CG}$ (red), $\mathrm{CHG}$ (green), and $\mathrm{CHH}$ (blue) from published data in wild type (Col) (top) or kyp suvh5 suvh6 (middle) (Stroud et al. 2013). A kyp suvh5 suvh6 minus wild type differential ( $\Delta$ DNA methylation) plot is also shown (bottom). Vertical dotted lines indicate the position of the centromere assembly gaps and vertical solid lines indicate telomeres. The pericentromeres, defined by higher than average DNA methylation, are indicated by light blue shading. (B) Plots showing $\log _{2}$ (SPO11-1-oligos/gDNA) (SPO11-1-oligos) in wild type (Col, black) and kyp suvh5 suvh6 (red) (top), in addition to the kyp suvh5 suvh6-wild type differential ( $\Delta$ SPO11-1-oligos) (middle), which is also overlaid with the $\Delta \mathrm{DNA}$ methylation from $A$ (bottom). Plots are annotated as in $A$. (C) Plots of the $A$. thaliana chromosomes showing the density of CEN180 repeats on forward (black) and reverse (red) strands. The TAIR10 centromeric assembly gaps are shown by the dotted blue line. The plots on the left show whole chromosomes, whereas the plots on the right show a close-up of the regions surrounding the centromere assembly gaps. (D) The density of log 2 (SPO11-1-oligos/gDNA) (SPO11-1-oligos) was analyzed in $\pm 10-\mathrm{kb}$ windows surrounding matches to the CEN180 consensus in wild type (black) or kyp suvh5 suvh6 (blue). The lower plot corresponds to a blow-up of a 1-kb window around the center of the upper plot. The lower plot highlights the approximate positions of CEN180 repeats using arrows and red dotted lines. Also shown is an identical analysis performed for the same number of randomly chosen sites.

containing Ds "launch-pads," triggering $D$ s transposition in clonal cell lineages within $F_{1}$ plants (Sundaresan et al. 1995). As Ds elements are known to preferentially transpose to linked sites (Jones et al. 1990; Bancroft and Dean 1993), a positive-negative selection scheme was implemented in $\mathrm{F}_{2}$ progeny to select against the Ds launch-pad and for transposed Ds (Sundaresan et al. 1995). In this way, recovery of $F_{2}$ transpositions was dependent on recombination between the transposed $D s$ and the launchpad and should be mostly unlinked.
Three launch-pads on Chromosome 1, DsE2, DsE3, and DsE6 (Fig. 6), were used to generate 9622 independent transpositions, which were mapped using TAIL-PCR (Springer et al. 1995). Unexpectedly, we recovered a dramatic enrichment of homozygous and heterozygous insertions into and nearby the CMT3 locus (214 out of 4044 insertions in total), but only when Ds elements were launched from a closely linked ( $360 \mathrm{~kb})$ centromere-proximal locus on Chromosome 1 (DsE3) and not from a centromere-distal locus (DeE6) located a comparable distance 


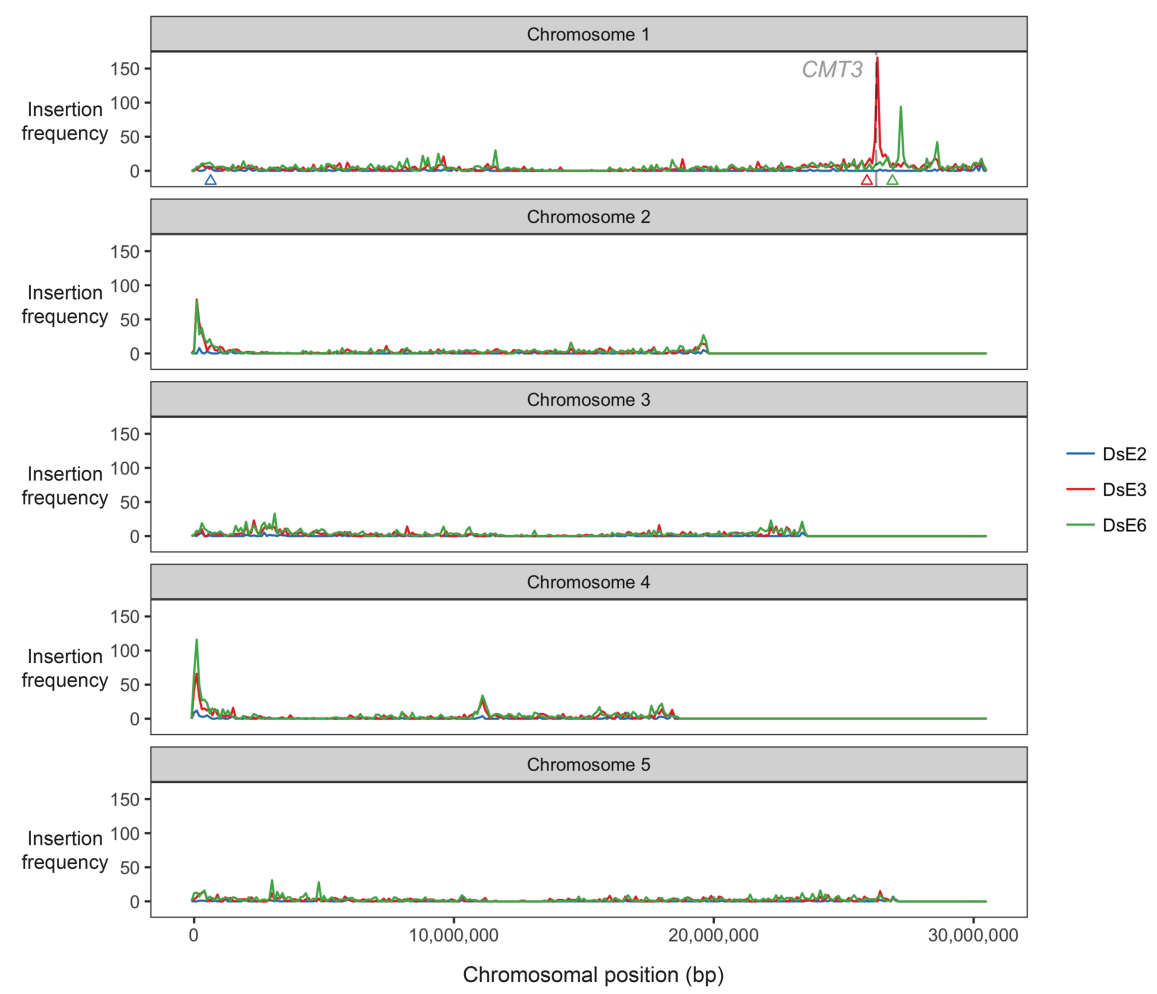

Figure 6. Transposon insertions in CMT3 appear to induce recombination. Enhancer trap DNA transposons ( $D S E)$ based on the maize transposable element Dissociation $(D s)$ were introduced into the A. thaliana genome on a T-DNA with a negative selectable marker (Sundaresan et al. 1995). Three independent DsE launch-pads were mapped to Chromosome 1: DsE2 (633,819 bp, blue triangle), DsE3 $(25,893,665 \mathrm{bp}$, red triangle), and DsE6 (26,877,449 bp, green triangle). After introducing Activator transposase, 9622 transpositions of DsE were isolated in $\mathrm{F}_{2}$ progeny by selecting for the transposon, but against the launch-pad, to select against linked transpositions which would otherwise be highly favored. New DsE insertions were mapped by sequencing flanking DNA. Transpositions from each Chromosome 1 launch-pad are displayed in 100-kb bins, and most were unlinked, including two hotspots corresponding to nucleolar organizer regions at the ends of Chromosomes 2 and 4 . However, two much sharper insertions hotspots, on Chromosome 1, were specific for closely linked launchpads, DsE3 (red triangle) and DsE6 (green triangle), respectively. The hotspot immediately distal to DSE3 ( $\sim \%$ of transpositions launched from DsE3) was in and immediately around the CMT3 gene $(26,248,318-26,253,585 \mathrm{bp})$.

from CMT3 ( 624 kb) (Fig. 6). One possible explanation was that flowers carrying homozygous $\mathrm{cm} t 3$ insertions arose by transposition into CMT3, followed by imprecise excision and mitotic recombination. Subsequent meiotic recombination between heterozygous transposed $D$ s elements and the launch-pad might be enhanced in $c m t 3$ anthers, leading to increased recovery of $\mathrm{F}_{2}$ "transposant" progeny carrying insertions near CMT3. Ac elements in maize and $D s$ elements in $A$. thaliana can stimulate mitotic recombination between flanking repeats (Athma and Peterson 1991; Xiao et al. 2000), but the excision/recombination scenario envisioned here has not been previously observed. Further work will be required to investigate the role of mitotic and meiotic recombination in the enhanced recovery of $D s$ insertions near $C M T 3$, and the extent that this might be caused by disruption of the non-CG DNA methylation/H3K9me2 pathway is currently unknown.

\section{Discussion}

Meiotic crossover in proximity to centromeres has been associated with chromosome missegregation and aneuploidy in fungi and animals (Koehler et al. 1996; Lamb et al. 1996; Rockmill et al. 2006). Hence, suppression of crossovers within centromeres is thought to play an important role in maintaining fidelity of genome transmission during meiosis. However, abundant evidence for recombinationassociated polymorphism in centromeric repeats exists, consistent with the effects of replication slippage, unequal crossover, and gene conversion (Ma and Bennetzen 2006; Nambiar and Smith 2016; Wolfgruber et al. 2016). For example, maize centromeric CRM retrotransposons have been observed to undergo meiotic gene conversion but not crossover (Shi et al. 2010), which indicates initiation of meiotic DSBs but downstream inhibition of recombination leading to crossover. We also observe evidence for SPO11-1-dependent DSBs within the $A$. thaliana CEN180 repeats, meaning that meiotic recombination may contribute to polymorphism within centromeric satellite repeat arrays in this species (Ito et al. 2007; Maheshwari et al. 2017).

As centromeric DSBs increase in kyp suvh5 suvh6 mutants, this demonstrates that epigenetic information, including H3K9me2 and non-CG DNA methylation, plays important roles in suppressing initiation of meiotic recombination in these regions. However, our data also reveal complexity in how chromatin shapes meiotic recombination around plant centromeres. First, while the genetically defined centromeric regions show increased DSBs in kyp suvh5 suvh6, in cmt3 we observed that crossovers were most elevated in adjacent pericentromeric regions. An important distinction between these experiments is that SPO11-1-oligos were mapped in a Col/Col homozygous background, whereas mapping crossover necessitates use of polymorphic Col/Ler hybrids. In a Col/ Ler hybrid context, centromeric crossovers are likely to be additionally suppressed by structural polymorphisms. For example, the inhibitory effect of structural polymorphism is evident within the $11.17-\mathrm{Mb}$ heterochromatic knob inversion on Chromosome 4, which is suppressed for crossovers in both wild-type and cmt3 populations (Fig. 2D; Fransz et al. 2000, 2016). Following DSB formation, resection occurs to generate $3^{\prime}$ single-stranded DNA that can perform strand invasion of homologous chromosomes (Keeney and Neale 2006). Inter-homolog recombination downstream from strand invasion is sensitive to heterology between the recombining chromosomes, which can have a local inhibitory effect on crossover formation and instead promote noncrossover repair and gene conversion (Dooner 1986; Borts and Haber 1987). A thaliana centromeric regions exhibit extensive structural variation, including within Gypsy retroelements (Kumekawa et al. 2000; Ito et al. 2007; Quadrana et al. 2016; Stuart et al. 2016), which may therefore suppress centromeric crossover formation downstream from inter-homolog strand invasion, despite

\section{Genome Research}

www.genome.org 
activation of meiotic DSBs in non-CG/H3K9me2 mutants. It is also possible that additional chromatin or epigenetic features enriched within the centromeres suppress crossover repair. For example, the kinetochore, CENH3 nucleosomes, or further heterochromatic marks such as H2A.W may be differentially enriched within the centromeric versus pericentromeric regions and cause inhibition of crossover maturation (Yelagandula et al. 2014; Vincenten et al. 2015).

An important question raised by our study is why pericentromeric crossover frequency increases in the H3K9me2/non-CG pathway mutants reported here, but not in met 1 and $d d m 1$ where reduced pericentromeric crossover frequency is observed (ColoméTatché et al. 2012; Melamed-Bessudo and Levy 2012; Mirouze et al. 2012; Yelina et al. 2012, 2015), despite both kyp suvh5 suvh6 and met1 showing increased SPO11-1-oligos in proximity to centromeres (Choi et al. 2018). MET1 and DDM1 play major roles in the maintenance of DNA methylation in the CG context. However, their molecular roles in other respects are distinct: (1) Non-CG DNA methylation is reduced in $d d m 1$ to a greater extent than met1 (Stroud et al. 2013); (2) gene body methylation is eliminated in met1 but not $d d m 1$ (Stroud et al. 2013); and (3) H3K9me2 is reduced more strongly in $d d m 1$ compared with met1 (Gendrel et al. 2002; Deleris et al. 2012). Therefore, we postulate that their common feature, loss of CG methylation within heterochromatin, alters progression and maturation of the meiotic recombination pathway, such that crossovers are favored in the chromosome arms, at the expense of the pericentromeres (Colomé-Tatché et al. 2012; Melamed-Bessudo and Levy 2012; Mirouze et al. 2012; Yelina et al. 2012, 2015). In contrast, H3K9me2/non-CG pathway mutants activate recombination in the heterochromatic regions such that maturation of crossovers in the pericentromeres is increased. Indeed, distinctions in recombination phenotype are consistent with the different effects on transcription, transposition, and chromosomal conformation associated with loss of CG versus non-CG DNA methylation maintenance pathways (Miura et al. 2001; Singer et al. 2001; Kato et al. 2003; Lippman et al. 2004; Zhang et al. 2006; Henderson and Jacobsen 2008; Lister et al. 2008; Colomé-Tatché et al. 2012; Melamed-Bessudo and Levy 2012; Mirouze et al. 2012; Stroud et al. 2012, 2013, 2014; Feng et al. 2014; Yelina et al. 2015).

We propose that, while both CG and non-CG DNA methylation inhibit centromeric meiotic DSBs (Choi et al. 2018), only non-CG methylation and/or H3K9me2 inhibit crossovers. In agreement with this idea, euchromatic crossover hotspots in A. thaliana can be silenced by RNA-directed DNA methylation associated with gain of $\mathrm{H} 3 \mathrm{~K} 9 \mathrm{me} 2$ and both CG and non-CG methylation (Yelina et al. 2015). Furthermore, in fission yeast, arrested recombination intermediates accumulate strongly in wild-type heterochromatin, but not in clr4 and rik1 (Recombination In K) heterochromatin, which lose H3K9me2 and undergo mitotic and meiotic recombination (Ellermeier et al. 2010; Zaratiegui et al. 2011). Mouse dnmt3l mutants also have altered DNA methylation and chromatin signatures and increased DSB initiation within retrotransposons, which is associated with meiotic catastrophe and infertility (Zamudio et al. 2015). In contrast, $A$. thaliana H3K9me2 and DNA methylation mutants are fully fertile, despite increased recombination initiation in the centromeric regions, suggesting that increased meiotic DSBs in transposons do not, per se, cause infertility. Suppression of heterochromatic recombination is a major barrier to introducing genetic diversity in crop plants like maize and wheat, where the majority of the chromosome is composed of pericentromeric heterochromatin and yet contains important genetic variation in functional genes and traits (Gore et al. 2009; Choulet et al. 2014). Therefore, an exciting prospect will be to modulate H3K9me2 and non-CG DNA methylation to unlock pericentromeric crossovers in crop breeding programs.

\section{Methods}

\section{Plant material}

A. thaliana plants were grown under long-day conditions $(16 \mathrm{~h}$ light $/ 8 \mathrm{~h}$ dark, at $150 \mu \mathrm{mol}$ light intensity) at $20^{\circ} \mathrm{C}$. We used the following mutant alleles: kyp-6 (SALK_041474) (Chan et al. 2006), cmt3-11 (SALK_148381) (Chan et al. 2006), cmt3-7 (Lindroth et al. 2001), kyp suvh5 suvh6 (SALK_041474, GK263C05, SAIL_1244_F04) (Johnson et al. 2008), drm1-2 drm2-2 (SALK_031705, SALK_150863) (Chan et al. 2006), drm1-2 drm2-2 cmt3-11 (SALK_031705, SALK_150863, SALK_148381) (Chan et al. 2006), cmt2-3 (SALK_012874) (Stroud et al. 2014), ddm1 (SALK_000590), zip4-2 (SALK_068052) (Chelysheva et al. 2007), and fancm-1 (EMS point mutant) (Crismani et al. 2012). The centromeric FTLS CTL5.11 and LTL5.4 were obtained from the Traffic line population (Wu et al. 2015).

\section{Mapping of $D s$ insertion sites}

Generation of the $D s$ transposant lines was previously reported. $D s$ insertion sites were amplified by TAIL-PCR (Springer et al. 1995; Sundaresan et al. 1995).

\section{Measuring crossovers using fluorescent pollen and seed}

Crossover scoring using the pollen FTL CEN3 was performed by flow cytometry, as previously reported (Yelina et al. 2013). Crossover scoring using seed FTLs $(420$, CTL5.11, LTL5.4) was performed by fluorescent imaging, as previously reported (Ziolkowski et al. 2015). Statistical analysis of fluorescent count data was performed as described (Yelina et al. 2015; Ziolkowski et al. 2015).

\section{Genotyping-by-sequencing}

Illumina sequencing libraries were constructed in 96-well format, as previously reported (Rowan et al. 2015; Yelina et al. 2015), with the following minor modifications. DNA was extracted from three rosette leaves of 5-wk-old plants and $150 \mathrm{ng}$ of DNA used as input for each library. DNA shearing was carried out for $20 \mathrm{~min}$ at $37^{\circ} \mathrm{C}$ with 0.4 units of DNA Shearase (Zymo Research). Each set of 96 libraries was sequenced on an Illumina NextSeq 500 (300-cycle Mid Output run). Sequencing data was analyzed to identify crossovers as previously reported, using the TIGER pipeline (Rowan et al. 2015; Yelina et al. 2015). Three hundred eightyfour cmt3-11 $\times c m t 3-7 \mathrm{~F}_{2}$ individuals were sequenced. Wild-type crossovers (CO) were mapped by sequencing $245 \mathrm{Col} \times$ Ler $\mathrm{F}_{2}$ individuals, which were combined with data from $192 \mathrm{~F}_{2}$ individuals (Choi et al. 2016).

The coordinates of crossover intervals called by TIGER were used for subsequent analysis. Centromeres were genetically defined as contiguous regions flanking the TAIR10 centromeric assembly gap that show an absence of crossovers in wild type (Copenhaver et al. 1999; Giraut et al. 2011; Salomé et al. 2012). We define the pericentromeric regions as regions flanking the centromeres with higher than chromosome average levels of DNA methylation. The euchromatic arms constitute the remainder of the chromosomes, from the telomeres to the pericentromeres (Supplemental Table S9). Crossovers (midpoints) were counted in these regions and compared to those expected at random according 
to physical distance and $\chi^{2}$ tests performed (Supplemental Table S10).

For chromosomal plots of crossovers and DNA methylation, crossovers were tallied in $10-\mathrm{kb}$ windows along the chromosomes, and crossover/10-kb values were then divided by the number of $\mathrm{F}_{2}$ individuals analyzed for each genotype. For DNA methylation, mean values were calculated in $10-\mathrm{kb}$ windows. Finally, for both crossovers and DNA methylation data, a rolling mean calculation was applied to smooth the data prior to plotting using the R function filter (R Core Team 2012). The crossover and DNA methylation differentials were calculated by subtracting wild-type from cmt3 values.

For telomere-centromere analysis, chromosome arms were first oriented such that each began at the telomere and ended at the centromere. The arms were then divided into windows representing $1 \%$ of their proportional lengths and crossovers assigned to these windows. Crossover values were divided by the number of $\mathrm{F}_{2}$ individuals analyzed for each genotype. These values were averaged across all chromosome arms and then plotted along the telomere-centromere axis with smoothing applied using the $\mathrm{R}$ function smooth.spline. For DNA methylation analysis along telomere-centromere axes, values were first calculated in 10-kb windows along the chromosomes. Chromosome arms were then oriented such that each began at the telomere and ended at the centromere and divided into windows representing $0.5 \%$ of their proportional lengths. Methylation values were then averaged across all chromosome arms and plotted along the telomere-centromere axis.

\section{SPO11-1-oligonucleotide sequencing}

A detailed protocol and analysis methodology are provided in an accompanying manuscript (Choi et al. 2018). A complementing SPO11-1-Myc spo11-1 line was crossed with kyp suvh5 suvh6 triple mutants and SPO11-1-Myc spo11-1 kyp suvh5 suvh6 plants identified for analysis. The CEN180 consensus sequence (5'-AACCTTC TTCTTGCTTCTCAAAGCTTTCATGGTGTAGCCAAAGTCCATATG AGTCTTTGGCTTTGTGTCTTCTAACAAGGAAACACTACTTAGGC TTTTAAGATCCGGTTGCGGTTTAAGTTCTTATACTCAATCATAC ACATGACATCAAGTCATATTCGACTCCAAAACACTAACC-3') was matched to the TAIR10 reference sequence using the $\mathrm{R}$ function matchPattern with max.mismatch set to 90 . The coverage value of normalized SPO11-1-oligonucleotides was analyzed in 20-kb windows around CEN180 matches and compared with analysis of the same number of randomly chosen positions.

\section{Meiotic immunocytology}

Chromosome spreads of $A$. thaliana pollen mother cells and immunostaining of ASY1 and H3K9me2 were performed using fresh buds, as described (Armstrong et al. 2002). Immunostaining of MLH1 was performed on acetic acid chromosome spreads on fixed buds, as described (Chelysheva et al. 2010). The following antibodies were used: $\alpha$-ASY1 (rabbit, 1/500 dilution, gift from Chris Franklin [University of Birmingham]) (Armstrong et al. 2002), $\alpha$ H3K9me2 (mouse, 1/200 dilution, Abcam, ab1220), and $\alpha$-MLH1 (rabbit, 1/200 dilution, gift from Mathilde Grelon [INRA, Versailles]) (Chelysheva et al. 2010). Microscopy was conducted using a DeltaVision Personal DV microscope (Applied Precision/GE Healthcare) equipped with a CDD CoolSNAP HQ2 camera (Photometrics). Image capture was performed using softWoRx software version 5.5 (Applied Precision/GE Healthcare). All slides within an experiment (e.g., Col, cmt3, and kyp suvh5 suvh6) were prepared alongside one another and images captured using the same exposure time. The staining pattern of ASY1 and DNA was used to iden- tify cells at leptotene, zygotene, or pachytene stage (SanchezMoran et al. 2007). Wild-type (Col) cells were first analyzed and a threshold pixel intensity value identified that removed background signal. This threshold was then applied to all images prior to further processing. Individual cell images were acquired as Zstacks of 16 optical sections of $0.25 \mu \mathrm{m}$ each, and the maximum intensity projection of the cell was reconstructed using ImageJ, as described (Lambing et al. 2015). The boundaries of each cell were manually defined and the total signal intensity within the cell measured. An adjacent region outside of the cell was used to measure mean background intensity and this value used to subtract from the within-cell intensity. The same methods were used for analysis of meiotic and somatic cells.

\section{Data access}

All data from this study have been submitted to ArrayExpress (https://www.ebi.ac.uk/arrayexpress/): SPO11-1-oligonucleotide sequencing data in wild type and kyp suvh5 suvh6 under accession number E-MTAB-5041; control libraries for SPO11-1-oligonucleotide sequencing under accession number E-MTAB-6257; and GBS crossover data from wild type and cmt3 populations under accession numbers E-MTAB-4657, E-MTAB-5476, and E-MTAB-6577 (GBS).

\section{Acknowledgments}

We thank Gregory Copenhaver (University of North Carolina, Chapel Hill), Avi Levy (The Weizmann Institute), and Scott Poethig (University of Pennsylvania) for fluorescent tagged lines, Raphaël Mercier (INRA, Versailles) for zip4-2 fancm-1, Steven Jacobsen (University of California, Los Angeles) for cmt3-7, Chris Franklin (University of Birmingham) for $\alpha$-ASY1, Mathilde Grelon (INRA, Versaille) for $\alpha$-MLH1, and the Gurdon Institute for access to microscopes. This work was supported by a William Miller Fellowship from the Watson School of Biological Sciences (C.J.U.), the Howard Hughes Medical Institute (R.A.M.), the Gordon and Betty Moore Foundation, as well as grants from the Gatsby Charitable Foundation (GAT2962 to I.R.H.), the Royal Society (University Research Fellowship to I.R.H.), BBSRC Meiogenix Industrial Partnership Award (BB/N007557/1 to I.R.H.), and by grants from the National Institutes of Health (R01GM067014) and from the National Science Foundation (R.A.M.).

Author contributions: C.J.U., I.R.H., and R.A.M. designed the study. C.J.U. performed all experiments apart from SPO11-1 experiments (K.C.), cytological analysis (C.L.), and construction of 171 of the wild-type genotyping-by-sequencing libraries (H.S.). Data analysis was performed by C.J.U., C.L., K.C., X.Z., H.S., F.B., J.S., E.E., Y.J., I.R.H., and R.A.M. The manuscript was prepared by C.J.U., I.R.H., and R.A.M.

\section{References}

Allshire RC, Ekwall K. 2015. Epigenetic regulation of chromatin states in Schizosaccharomyces pombe. Cold Spring Harb Perspect Biol 7: a018770.

Allshire RC, Karpen GH. 2008. Epigenetic regulation of centromeric chromatin: old dogs, new tricks? Nat Rev Genet 9: 923-937.

Armstrong SJ, Franklin FC, Jones GH. 2001. Nucleolus-associated telomere clustering and pairing precede meiotic chromosome synapsis in Arabidopsis thaliana. J Cell Sci 114: 4207-4217.

Armstrong SJ, Caryl AP, Jones GH, Franklin FCH. 2002. Asy1, a protein required for meiotic chromosome synapsis, localizes to axis-associated chromatin in Arabidopsis and Brassica. J Cell Sci 115: 3645-3655.

Athma P, Peterson T. 1991. Ac induces homologous recombination at the maize P locus. Genetics 128: 163-173.

\section{Genome Research}

www.genome.org 
Bancroft I, Dean C. 1993. Transposition pattern of the maize element Ds in Arabidopsis thaliana. Genetics 134: 1221-1229.

Bartee L, Malagnac F, Bender J. 2001. Arabidopsis cmt3 chromomethylase mutations block non-CG methylation and silencing of an endogenous gene. Genes Dev 15: 1753-1758.

Berchowitz LE, Copenhaver GP. 2008. Fluorescent Arabidopsis tetrads: a visual assay for quickly developing large crossover and crossover interference data sets. Nat Protoc 3: 41-50.

Borts RH, Haber JE. 1987. Meiotic recombination in yeast: alteration by multiple heterozygosities. Science 237: 1459-1465.

Cao X, Jacobsen SE. 2002. Role of the Arabidopsis DRM methyltransferases in de novo DNA methylation and gene silencing. Curr Biol 12: 1138-1144.

Cao X, Aufsatz W, Zilberman D, Mette MF, Huang MS, Matzke M, Jacobsen SE. 2003. Role of the DRM and CMT3 methyltransferases in RNA-directed DNA methylation. Curr Biol 13: 2212-2217.

Chan SW-L, Henderson IR, Zhang X, Shah G, Chien JS-C, Jacobsen SE. 2006. RNAi, DRD1, and histone methylation actively target developmentally important non-CG DNA methylation in Arabidopsis. PLoS Genet 2: e83.

Chelysheva L, Gendrot G, Vezon D, Doutriaux M-P, Mercier R, Grelon M. 2007. Zip4/Spo22 is required for class I CO formation but not for synapsis completion in Arabidopsis thaliana. PLoS Genet 3: e83.

Chelysheva L, Grandont L, Vrielynck N, le Guin S, Mercier R, Grelon M. 2010. An easy protocol for studying chromatin and recombination protein dynamics during Arabidopsis thaliana meiosis: immunodetection of cohesins, histones and MLH1. Cytogenet Genome Res 129: 143-153.

Choi K, Henderson IR. 2015. Meiotic recombination hotspots - a comparative view. Plant J 83: 52-61.

Choi K, Zhao X, Kelly KA, Venn O, Higgins JD, Yelina NE, Hardcastle TJ Ziolkowski PA, Copenhaver GP, Franklin FCH, et al. 2013. Arabidopsis meiotic crossover hot spots overlap with H2A.Z nucleosomes at gene promoters. Nat Genet 45: 1327-1336.

Choi K, Reinhard C, Serra H, Ziolkowski PA, Underwood CJ, Zhao X, Hardcastle TJ, Yelina NE, Griffin C, Jackson M, et al. 2016. Recombination rate heterogeneity within Arabidopsis disease resistance genes. PLoS Genet 12: e1006179.

Choi K, Zhao X, Tock AJ, Lambing C, Underwood CJ, Hardcastle TJ, Serra H, Kim J, Cho HS, Kim J, et al. 2018. Nucleosomes and DNA methylation shape meiotic DSB frequency in Arabidopsis thaliana transposons and gene regulatory regions. Genome Res (this issue). doi: 10.1101/ gr.225599.117.

Choulet F, Alberti A, Theil S, Glover N, Barbe V, Daron J, Pingault L, Sourdille P, Couloux A, Paux E, et al. 2014. Structural and functional partitioning of bread wheat chromosome 3B. Science 345: 1249721.

Colomé-Tatché M, Cortijo S, Wardenaar R, Morgado L, Lahouze B, Sarazin A, Etcheverry M, Martin A, Feng S, Duvernois-Berthet E, et al. 2012. Features of the Arabidopsis recombination landscape resulting from the combined loss of sequence variation and DNA methylation. Proc Natl Acad Sci 109: 16240-16245.

Copenhaver GP, Nickel K, Kuromori T, Benito MI, Kaul S, Lin X, Bevan M, Murphy G, Harris B, Parnell LD, et al. 1999. Genetic definition and sequence analysis of Arabidopsis centromeres. Science 286: 2468-2474.

Crismani W, Girard C, Froger N, Pradillo M, Santos JL, Chelysheva L, Copenhaver GP, Horlow C, Mercier R. 2012. FANCM limits meiotic crossovers. Science 336: 1588-1590.

De Massy B. 2013. Initiation of meiotic recombination: how and where? Conservation and specificities among eukaryotes. Annu Rev Genet 47: 563-599.

Deleris A, Stroud H, Bernatavichute Y, Johnson E, Klein G, Schubert D, Jacobsen SE. 2012. Loss of the DNA methyltransferase MET1 induces H3K9 hypermethylation at PcG target genes and redistribution of H3K27 trimethylation to transposons in Arabidopsis thaliana. PLoS Genet 8: e1003062.

Dooner HK. 1986. Genetic fine structure of the BRONZE locus in maize. Genetics 113: 1021-1036.

Du J, Zhong X, Bernatavichute YV, Stroud H, Feng S, Caro E, Vashisht AA Terragni J, Chin HG, Tu A, et al. 2012. Dual binding of chromomethylase domains to H3K9me2-containing nucleosomes directs DNA methylation in plants. Cell 151: $167-180$.

Du J, Johnson LM, Groth M, Feng S, Hale CJ, Li S, Vashisht AA, GallegoBartolome J, Wohlschlegel JA, Patel DJ, et al. 2014. Mechanism of DNA methylation-directed histone methylation by KRYPTONITE. Mol Cell 55: 495-504.

Dubin MJ, Zhang P, Meng D, Remigereau M-S, Osborne EJ, Paolo Casale F, Drewe P, Kahles A, Jean G, Vilhjálmsson B, et al. 2015. DNA methylation in Arabidopsis has a genetic basis and shows evidence of local adaptation. eLife 4: e05255.

Ebbs ML, Bender J. 2006. Locus-specific control of DNA methylation by the Arabidopsis SUVH5 histone methyltransferase. Plant Cell 18: 1166-1176.
Ellermeier C, Higuchi EC, Phadnis N, Holm L, Geelhood JL, Thon G, Smith GR. 2010. RNAi and heterochromatin repress centromeric meiotic recombination. Proc Natl Acad Sci 107: 8701-8705.

Feng S, Cokus SJ, Schubert V, Zhai J, Pellegrini M, Jacobsen SE. 2014. Genome-wide Hi-C analyses in wild-type and mutants reveal high-resolution chromatin interactions in Arabidopsis. Mol Cell 55: 694-707.

Fransz PF, Armstrong S, de Jong JH, Parnell LD, van Drunen C, Dean C, Zabel $\mathrm{P}$, Bisseling T, Jones GH. 2000. Integrated cytogenetic map of chromosome arm $4 \mathrm{~S}$ of $A$. thaliana: structural organization of heterochromatic knob and centromere region. Cell 100: 367-376.

Fransz P, Linc G, Lee C-R, Aflitos SA, Lasky JR, Toomajian C, Hoda A, Peters J, van Dam P, Ji X, et al. 2016. Molecular, genetic and evolutionary analysis of a paracentric inversion in Arabidopsis thaliana. Plant $J$ 88: $159-178$.

Gendrel A-V, Lippman Z, Yordan C, Colot V, Martienssen RA. 2002. Dependence of heterochromatic histone H3 methylation patterns on the Arabidopsis gene DDM1. Science 297: 1871-1873.

Giraut L, Falque M, Drouaud J, Pereira L, Martin OC, Mézard C. 2011. Genome-wide crossover distribution in Arabidopsis thaliana meiosis reveals sex-specific patterns along chromosomes. PLoS Genet 7: e1002354.

Gore MA, Chia J-M, Elshire RJ, Sun Q, Ersoz ES, Hurwitz BL, Peiffer JA, McMullen MD, Grills GS, Ross-Ibarra J, et al. 2009. A first-generation haplotype map of maize. Science 326: 1115-1117.

Grelon M, Vezon D, Gendrot G, Pelletier G. 2001. AtSPO11-1 is necessary for efficient meiotic recombination in plants. EMBO J 20: 589-600.

Hartung F, Wurz-Wildersinn R, Fuchs J, Schubert I, Suer S, Puchta H. 2007. The catalytically active tyrosine residues of both SPO11-1 and SPO11-2 are required for meiotic double-strand break induction in Arabidopsis. Plant Cell 19: 3090-3099.

Hellsten U, Wright KM, Jenkins J, Shu S, Yuan Y, Wessler SR, Schmutz J, Willis JH, Rokhsar DS. 2013. Fine-scale variation in meiotic recombination in Mimulus inferred from population shotgun sequencing. Proc Nat Acad Sci 110: 19478-19482.

Henderson IR, Jacobsen SE. 2008. Tandem repeats upstream of the Arabidopsis endogene SDC recruit non-CG DNA methylation and initiate siRNA spreading. Genes Dev 22: 1597-1606.

Higgins JD, Armstrong SJ, Franklin FCH, Jones GH. 2004. The Arabidopsis MutS homolog AtMSH4 functions at an early step in recombination: evidence for two classes of recombination in Arabidopsis. Genes Dev 18: 2557-2570.

Inagaki S, Miura-Kamio A, Nakamura Y, Lu F, Cui X, Cao X, Kimura H, Saze H, Kakutani T. 2010. Autocatalytic differentiation of epigenetic modifications within the Arabidopsis genome. EMBO J 29: 3496-3506.

Ito H, Miura A, Takashima K, Kakutani T. 2007. Ecotype-specific and chromosome-specific expansion of variant centromeric satellites in Arabidopsis thaliana. Mol Genet Genomics 277: 23-30.

Jackson JP, Lindroth AM, Cao X, Jacobsen SE. 2002. Control of CpNpG DNA methylation by the KRYPTONITE histone H3 methyltransferase. Nature 416: $556-560$.

Johnson LM, Bostick M, Zhang X, Kraft E, Henderson I, Callis J, Jacobsen SE. 2007. The SRA methyl-cytosine-binding domain links DNA and histone methylation. Curr Biol 17: 379-384.

Johnson LM, Law JA, Khattar A, Henderson IR, Jacobsen SE. 2008. SRAdomain proteins required for DRM2-mediated de novo DNA methylation. PLoS Genet 4: e1000280.

Jones JD, Carland F, Lim E, Ralston E, Dooner HK. 1990. Preferential transposition of the maize element Activator to linked chromosomal locations in tobacco. Plant Cell 2: 701-707.

Kankel MW, Ramsey DE, Stokes TL, Flowers SK, Haag JR, Jeddeloh JA, Riddle NC, Verbsky ML, Richards EJ. 2003. Arabidopsis MET1 cytosine methyltransferase mutants. Genetics 163: 1109-1122.

Kato M, Miura A, Bender J, Jacobsen SE, Kakutani T. 2003. Role of CG and non-CG methylation in immobilization of transposons in Arabidopsis. Curr Biol 13: 421-426.

Kauppi L, Jeffreys AJ, Keeney S. 2004. Where the crossovers are: recombination distributions in mammals. Nat Rev Genet 5: 413-424.

Keeney S, Kleckner N. 1995. Covalent protein-DNA complexes at the 5' strand termini of meiosis-specific double-strand breaks in yeast. Proc Natl Acad Sci 92: 11274-11278.

Keeney S, Neale MJ. 2006. Initiation of meiotic recombination by formation of DNA double-strand breaks: mechanism and regulation. Biochem Soc Trans 34: 523-525.

Keeney S, Giroux CN, Kleckner N. 1997. Meiosis-specific DNA doublestrand breaks are catalyzed by Spo11, a member of a widely conserved protein family. Cell 88: 375-384.

Koehler KE, Boulton CL, Collins HE, French RL, Herman KC, Lacefield SM, Madden LD, Schuetz CD, Hawley RS. 1996. Spontaneous X chromosome MI and MII nondisjunction events in Drosophila melanogaster oocytes have different recombinational histories. Nat Genet 14: 406-414. 
Kumekawa N, Hosouchi T, Tsuruoka H, Kotani H. 2000. The size and sequence organization of the centromeric region of Arabidopsis thaliana chromosome 5. DNA Res 7: 315-321.

Lamb NE, Freeman SB, Savage-Austin A, Pettay D, Taft L, Hersey J, Gu Y, Shen J, Saker D, May KM, et al. 1996. Susceptible chiasmate configurations of chromosome 21 predispose to non-disjunction in both maternal meiosis I and meiosis II. Nat Genet 14: 400-405.

Lambie EJ, Roeder GS. 1988. A yeast centromere acts in cis to inhibit meiotic gene conversion of adjacent sequences. Cell 52: 863-873.

Lambing C, Osman K, Nuntasoontorn K, West A, Higgins JD, Copenhaver GP, Yang J, Armstrong SJ, Mechtler K, Roitinger E, et al. 2015. Arabidopsis PCH2 mediates meiotic chromosome remodeling and maturation of crossovers. PLoS Genet 11: e1005372.

Lange J, Yamada S, Tischfield SE, Pan J, Kim S, Zhu X, Socci ND, Jasin M, Keeney S. 2016. The landscape of mouse meiotic double-strand break formation, processing, and repair. Cell 167: 695-708.e16.

Lhuissier FGP, Offenberg HH, Wittich PE, Vischer NOE, Heyting C. 2007. The mismatch repair protein MLH1 marks a subset of strongly interfering crossovers in tomato. Plant Cell 19: 862-876.

Lindroth AM, Cao X, Jackson JP, Zilberman D, McCallum CM, Henikoff S, Jacobsen SE. 2001. Requirement of CHROMOMETHYLASE3 for maintenance of CpXpG methylation. Science 292: 2077-2080.

Lippman Z, Gendrel A-V, Black M, Vaughn MW, Dedhia N, McCombie WR, Lavine K, Mittal V, May B, Kasschau KD, et al. 2004. Role of transposable elements in heterochromatin and epigenetic control. Nature 430: 471-476.

Lister R, O'Malley RC, Tonti-Filippini J, Gregory BD, Berry CC, Millar AH, Ecker JR. 2008. Highly integrated single-base resolution maps of the epigenome in Arabidopsis. Cell 133: 523-536.

Ma J, Bennetzen JL. 2006. Recombination, rearrangement, reshuffling, and divergence in a centromeric region of rice. Proc Natl Acad Sci 103: 383-388.

Maheshwari S, Ishii T, Brown CT, Houben A, Comai L. 2017. Centromere location in Arabidopsis is unaltered by extreme divergence in CENH3 protein sequence. Genome Res 27: 471-478.

Malagnac F, Bartee L, Bender J. 2002. An Arabidopsis SET domain protein required for maintenance but not establishment of DNA methylation. EMBO J 21: 6842-6852.

Malik HS, Henikoff S. 2009. Major evolutionary transitions in centromere complexity. Cell 138: 1067-1082.

Maloisel L, Rossignol JL. 1998. Suppression of crossing-over by DNA methylation in Ascobolus. Genes Dev 12: 1381-1389.

Marí-Ordóñez A, Marchais A, Etcheverry M, Martin A, Colot V, Voinnet O. 2013. Reconstructing de novo silencing of an active plant retrotransposon. Nat Genet 45: 1029-1039.

Mayer KFX, Waugh R, Brown JWS, Schulman A, Langridge P, Platzer M, Fincher GB, Muehlbauer GJ, Sato K, Close TJ, et al. 2012. A physical, genetic and functional sequence assembly of the barley genome. Nature 491: 711-716.

McKinley KL, Cheeseman IM. 2015. The molecular basis for centromere identity and function. Nat Rev Mol Cell Biol 17: 16-29.

Melamed-Bessudo C, Levy AA. 2012. Deficiency in DNA methylation increases meiotic crossover rates in euchromatic but not in heterochromatic regions in Arabidopsis. Proc Natl Acad Sci 109: E981-E988.

Melamed-Bessudo C, Yehuda E, Stuitje AR, Levy AA. 2005. A new seed-based assay for meiotic recombination in Arabidopsis thaliana. Plant $J$ 43: $458-466$.

Mercier R, Jolivet S, Vezon D, Huppe E, Chelysheva L, Giovanni M, Nogué F, Doutriaux M-P, Horlow C, Grelon M, et al. 2005. Two meiotic crossover classes cohabit in Arabidopsis: One is dependent on MER3, whereas the other one is not. Curr Biol 15: 692-701.

Mirouze M, Reinders J, Bucher E, Nishimura T, Schneeberger K, Ossowski S, Cao J, Weigel D, Paszkowski J, Mathieu O. 2009. Selective epigenetic control of retrotransposition in Arabidopsis. Nature 461: 427-430.

Mirouze M, Lieberman-Lazarovich M, Aversano R, Bucher E, Nicolet J, Reinders J, Paszkowski J. 2012. Loss of DNA methylation affects the recombination landscape in Arabidopsis. Proc Natl Acad Sci 109: 5880-5885

Miura A, Yonebayashi S, Watanabe K, Toyama T, Shimada H, Kakutani T. 2001. Mobilization of transposons by a mutation abolishing full DNA methylation in Arabidopsis. Nature 411: 212-214.

Nagaki K, Talbert PB, Zhong CX, Dawe RK, Henikoff S, Jiang J. 2003. Chromatin immunoprecipitation reveals that the 180-bp satellite repeat is the key functional DNA element of Arabidopsis thaliana centromeres. Genetics 163: 1221-1225.

Nambiar M, Smith GR. 2016. Repression of harmful meiotic recombination in centromeric regions. Semin Cell Dev Biol 54: 188-197.

Pan J, Sasaki M, Kniewel R, Murakami H, Blitzblau HG, Tischfield SE, Zhu X, Neale MJ, Jasin M, Socci ND, et al. 2011. A hierarchical combination of factors shapes the genome-wide topography of yeast meiotic recombination initiation. Cell 144: 719-731.
Quadrana L, Bortolini Silveira A, Mayhew GF, LeBlanc C, Martienssen RA, Jeddeloh JA, Colot V. 2016. The Arabidopsis thaliana mobilome and its impact at the species level. eLife 5: e15716.

R Core Team. 2012. R: a language and environment for statistical computing. $\mathrm{R}$ Foundation for Statistical Computing, Vienna, Austria. http://www.Rproject.org/.

Reinders J, Wulff BBH, Mirouze M, Marí-Ordóñez A, Dapp M, Rozhon W, Bucher E, Theiler G, Paszkowski J. 2009. Compromised stability of DNA methylation and transposon immobilization in mosaic Arabidopsis epigenomes. Genes Dev 23: 939-950.

Robert T, Nore A, Brun C, Maffre C, Crimi B, Bourbon H-M, de Massy B. 2016. The TopoVIB-Like protein family is required for meiotic DNA double-strand break formation. Science 351: 943-949.

Rockmill B, Voelkel-Meiman K, Roeder GS. 2006. Centromere-proximal crossovers are associated with precocious separation of sister chromatids during meiosis in Saccharomyces cerevisiae. Genetics 174: 1745-1754.

Rowan BA, Patel V, Weigel D, Schneeberger K. 2015. Rapid and inexpensive whole-genome genotyping-by-sequencing for crossover localization and fine-scale genetic mapping. G3 (Bethesda) 5: 385-398.

Salomé PA, Bomblies K, Fitz J, Laitinen RAE, Warthmann N, Yant L, Weigel D. 2012. The recombination landscape in Arabidopsis thaliana F2 populations. Heredity (Edinb) 108: 447-455.

Sanchez-Moran E, Santos J-L, Jones GH, Franklin FCH. 2007. ASY1 mediates AtDMC1-dependent interhomolog recombination during meiosis in Arabidopsis. Genes Dev 21: 2220-2233.

Saze H, Mittelsten Scheid O, Paszkowski J. 2003. Maintenance of CpG methylation is essential for epigenetic inheritance during plant gametogenesis. Nat Genet 34: 65-69.

Serra H, Lambing C, Griffin CH, Topp SD, Nageswaran DC, Underwood CJ, Ziolkowski PA, Séguéla-Arnaud M, Fernandes JB, Mercier R, et al. 2018. Massive crossover elevation via combination of HEI1O and recq4a recq4b during Arabidopsis meiosis. Proc Natl Acad Sci doi: 10.1073/ pnas. 1713071115 .

Shi J, Wolf SE, Burke JM, Presting GG, Ross-Ibarra J, Dawe RK. 2010. Widespread gene conversion in centromere cores. PLoS Biol 8: e1000327.

Shilo S, Melamed-Bessudo C, Dorone Y, Barkai N, Levy AA. 2015. DNA crossover motifs associated with epigenetic modifications delineate open chromatin regions in Arabidopsis. Plant Cell 27: 2427-2436.

Singer T, Yordan C, Martienssen RA. 2001. Robertson's Mutator transposons in $A$. thaliana are regulated by the chromatin-remodeling gene Decrease in DNA Methylation (DDM1). Genes Dev 15: 591-602.

Springer PS, McCombie WR, Sundaresan V, Martienssen RA. 1995. Gene trap tagging of PROLIFERA, an essential MCM2-3-5-like gene in Arabidopsis. Science 268: 877-880.

Stroud H, Hale CJ, Feng S, Caro E, Jacob Y, Michaels SD, Jacobsen SE. 2012. DNA methyltransferases are required to induce heterochromatic re-replication in Arabidopsis. PLoS Genet 8: e1002808.

Stroud H, Greenberg MVC, Feng S, Bernatavichute YV, Jacobsen SE. 2013. Comprehensive analysis of silencing mutants reveals complex regulation of the Arabidopsis methylome. Cell 152: 352-364.

Stroud H, Do T, Du J, Zhong X, Feng S, Johnson L, Patel DJ, Jacobsen SE. 2014. Non-CG methylation patterns shape the epigenetic landscape in Arabidopsis. Nat Struct Mol Biol 21: 64-72.

Stuart T, Eichten S, Cahn J, Karpievitch Y, Borevitz J, Lister R. 2016. Population scale mapping of transposable element diversity reveals links to gene regulation and epigenomic variation. eLife 5: e20777.

Sundaresan V, Springer P, Volpe T, Haward S, Jones JD, Dean C, Ma H, Martienssen R. 1995. Patterns of gene action in plant development revealed by enhancer trap and gene trap transposable elements. Genes Dev 9: 1797-1810.

Szostak JW, Orr-Weaver TL, Rothstein RJ, Stahl FW. 1983. The doublestrand-break repair model for recombination. Cell 33: 25-35.

Teixeira FK, Heredia F, Sarazin A, Roudier F, Boccara M, Ciaudo C, Cruaud C, Poulain J, Berdasco M, Fraga MF, et al. 2009. A role for RNAi in the selective correction of DNA methylation defects. Science 323: 1600-1604.

Tsukahara S, Kawabe A, Kobayashi A, Ito T, Aizu T, Shin-i T, Toyoda A, Fujiyama A, Tarutani Y, Kakutani T. 2012. Centromere-targeted de novo integrations of an LTR retrotransposon of Arabidopsis lyrata. Genes Dev 26: 705-713.

Vincenten N, Kuhl L-M, Lam I, Oke A, Kerr AR, Hochwagen A, Fung J, Keeney S, Vader G, Marston AL. 2015. The kinetochore prevents centromere-proximal crossover recombination during meiosis. eLife 4: e10850.

Vrielynck N, Chambon A, Vezon D, Pereira L, Chelysheva L, De Muyt A, Mézard C, Mayer C, Grelon M. 2016. A DNA topoisomerase VI-like complex initiates meiotic recombination. Science 351: 939-943.

Wei F, Zhang J, Zhou S, He R, Schaeffer M, Collura K, Kudrna D, Faga BP, Wissotski M, Golser W, et al. 2009. The physical and genetic framework of the maize B73 genome. PLoS Genet 5: e1000715.

\section{Genome Research}

www.genome.org 
Westphal T, Reuter G. 2002. Recombinogenic effects of suppressors of position-effect variegation in Drosophila. Genetics 160: 609-621.

Wijnker E, Velikkakam James G, Ding J, Becker F, Klasen JR, Rawat V, Rowan BA, de Jong DF, de Snoo CB, Zapata L, et al. 2013. The genomic landscape of meiotic crossovers and gene conversions in Arabidopsis thaliana. eLife 2: e01426.

Wolfgruber TK, Nakashima MM, Schneider KL, Sharma A, Xie Z, Albert PS Xu R, Bilinski P, Dawe RK, Ross-Ibarra J, et al. 2016. High quality maize centromere 10 sequence reveals evidence of frequent recombination events. Front Plant Sci 7: 308.

Woo HR, Dittmer TA, Richards EJ. 2008. Three SRA-domain methylcytosine-binding proteins cooperate to maintain global CpG methylation and epigenetic silencing in Arabidopsis. PLoS Genet 4: e1000156.

Wu G, Rossidivito G, Hu T, Berlyand Y, Poethig RS. 2015. Traffic lines: new tools for genetic analysis in Arabidopsis thaliana. Genetics 200: 35-45.

Xiao YL, Li X, Peterson T. 2000. Ac insertion site affects the frequency of transposon-induced homologous recombination at the maize p1 locus. Genetics 156: 2007-2017.

Yelagandula R, Stroud H, Holec S, Zhou K, Feng S, Zhong X, Muthurajan UM, Nie X, Kawashima T, Groth M, et al. 2014. The histone variant H2A.W defines heterochromatin and promotes chromatin condensation in Arabidopsis. Cell 158: 98-109.

Yelina NE, Choi K, Chelysheva L, Macaulay M, de Snoo B, Wijnker E, Miller N, Drouaud J, Grelon M, Copenhaver GP, et al. 2012. Epigenetic remodeling of meiotic crossover frequency in Arabidopsis thaliana DNA methyltransferase mutants. PLoS Genet 8: e1002844.

Yelina NE, Ziolkowski PA, Miller N, Zhao X, Kelly KA, Muñoz DF, Mann DJ, Copenhaver GP, Henderson IR. 2013. High-throughput analysis of meiotic crossover frequency and interference via flow cytometry of fluorescent pollen in Arabidopsis thaliana. Nat Protoc 8: 2119-2134.
Yelina NE, Lambing C, Hardcastle TJ, Zhao X, Santos B, Henderson IR. 2015. DNA methylation epigenetically silences crossover hot spots and controls chromosomal domains of meiotic recombination in Arabidopsis. Genes Dev 29: 2183-2202.

Zamudio N, Barau J, Teissandier A, Walter M, Borsos M, Servant N, Bourc'his D. 2015. DNA methylation restrains transposons from adopting a chromatin signature permissive for meiotic recombination. Genes Dev 29: 1256-1270.

Zaratiegui M, Castel SE, Irvine DV, Kloc A, Ren J, Li F, de Castro E, Marín L, Chang A-Y, Goto D, et al. 2011. RNAi promotes heterochromatic silencing through replication-coupled release of RNA Pol II. Nature 479: 135-138.

Zemach A, Kim MY, Hsieh P-H, Coleman-Derr D, Eshed-Williams L, Thao K, Harmer SL, Zilberman D. 2013. The Arabidopsis nucleosome remodeler DDM1 allows DNA methyltransferases to access H1-containing heterochromatin. Cell 153: 193-205.

Zhang X, Yazaki J, Sundaresan A, Cokus S, Chan SW-L, Chen H, Henderson IR, Shinn P, Pellegrini M, Jacobsen SE, et al. 2006. Genome-wide highresolution mapping and functional analysis of DNA methylation in Arabidopsis. Cell 126: 1189-1201.

Ziolkowski PA, Berchowitz LE, Lambing C, Yelina NE, Zhao X, Kelly KA, Choi K, Ziolkowska L, June V, Sanchez-Moran E, et al. 2015. Juxtaposition of heterozygous and homozygous regions causes reciprocal crossover remodelling via interference during Arabidopsis meiosis. eLife 4: e03708.

Received July 3, 2017; accepted in revised form January 15, 2018. 


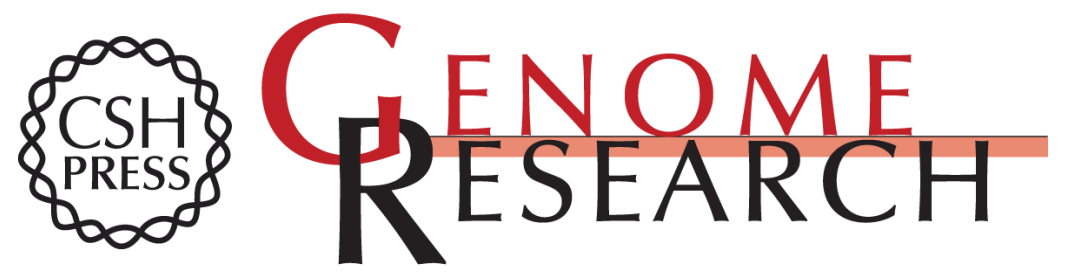

\section{Epigenetic activation of meiotic recombination near Arabidopsis thaliana centromeres via loss of $\mathrm{H} 3 \mathrm{~K} 9 \mathrm{me} 2$ and non-CG DNA methylation}

Charles J. Underwood, Kyuha Choi, Christophe Lambing, et al.

Genome Res. 2018 28: 519-531 originally published online March 12, 2018

Access the most recent version at doi:10.1101/gr.227116.117

\section{Supplemental http://genome.cshlp.org/content/suppl/2018/03/09/gr.227116.117.DC1 \\ Material}

Related Content

References

Open Access

Creative

Commons

License

Email Alerting
Service
Nucleosomes and DNA methylation shape meiotic DSB frequency in Arabidopsis thaliana transposons and gene regulatory regions Kyuha Choi, Xiaohui Zhao, Andrew J. Tock, et al.

Genome Res. April , 2018 28: 532-546

This article cites 119 articles, 53 of which can be accessed free at: http://genome.cshlp.org/content/28/4/519.full.html\#ref-list-1

Articles cited in:

http://genome.cshlp.org/content/28/4/519.full.html\#related-urls

Freely available online through the Genome Research Open Access option.

This article, published in Genome Research, is available under a Creative Commons License (Attribution 4.0 International), as described at http://creativecommons.org/licenses/by/4.0/.

Receive free email alerts when new articles cite this article - sign up in the box at the top right corner of the article or click here.

\section{Affordable, Accurate Sequencing.}

